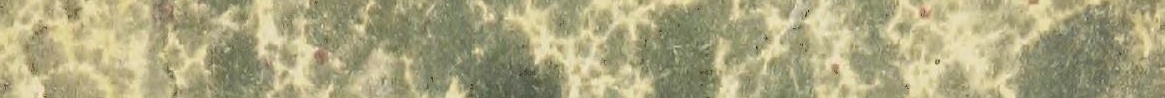
$32+24+2 \times 2 y+2$

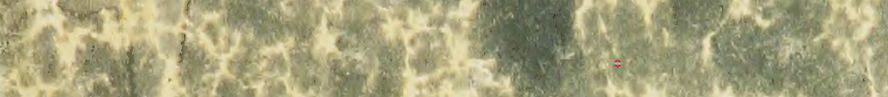
$H\left(x+2 y^{2}+2 x+x\right.$

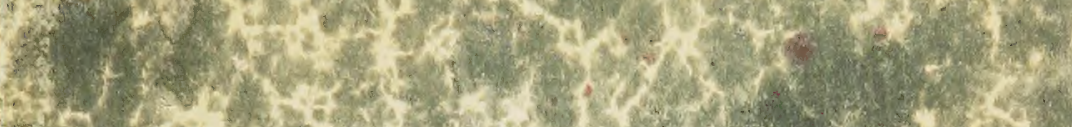

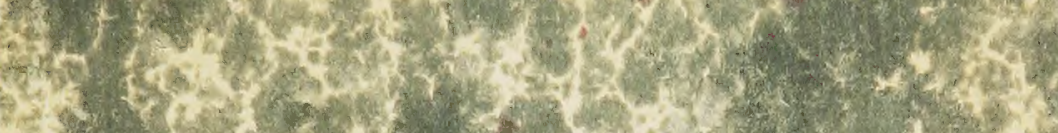

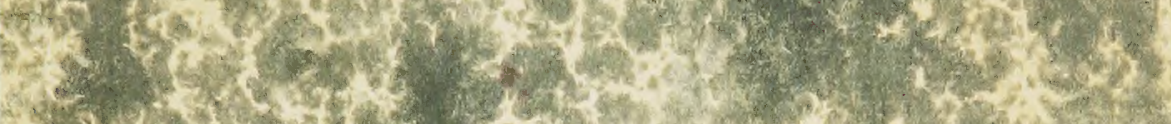
- 4

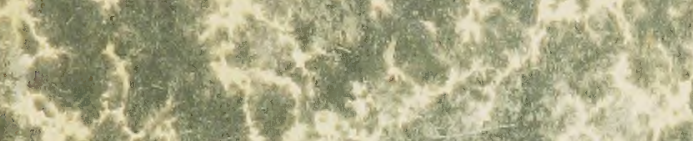
$x^{2}+x^{3}+x^{2}-1$ mox $10-5$

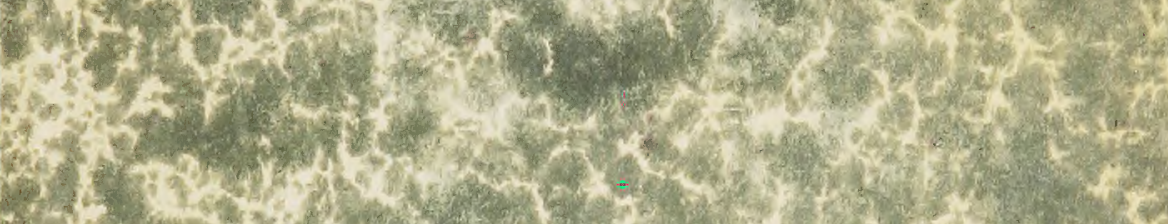

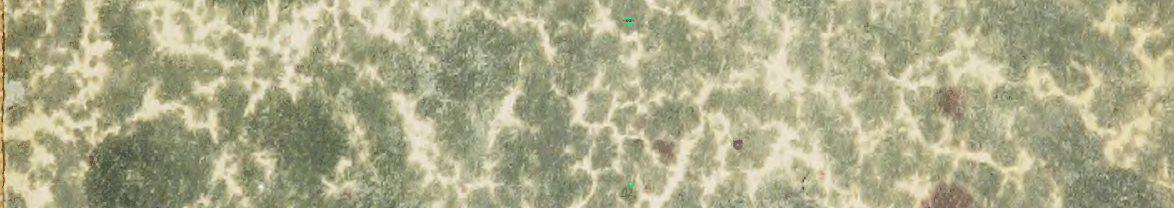

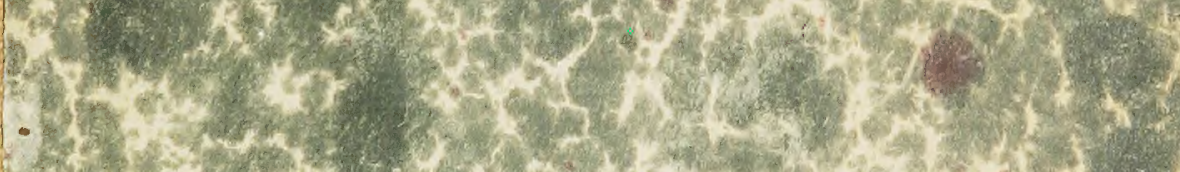

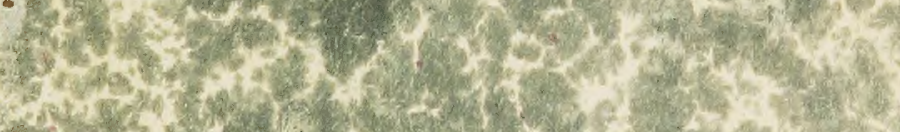
1.5. 3.

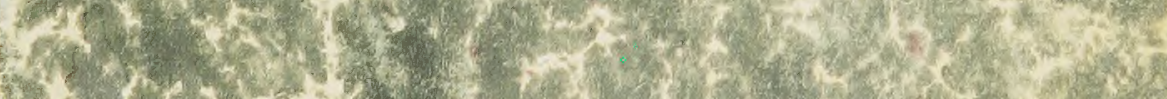

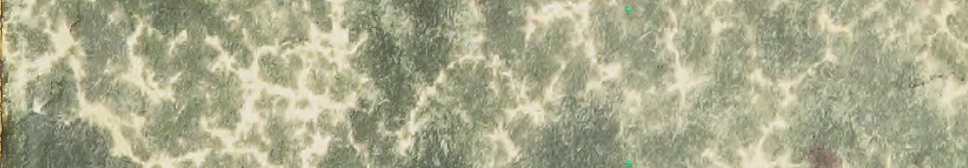

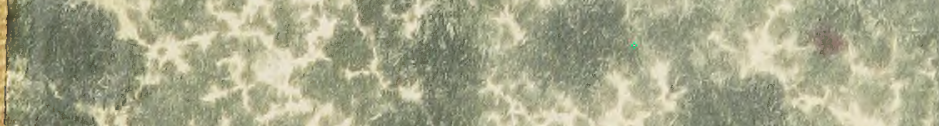

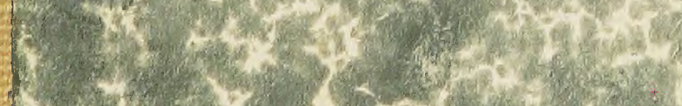

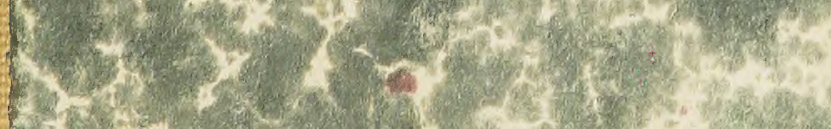
(x) 4 


\section{QL781 \\ $B 83$}

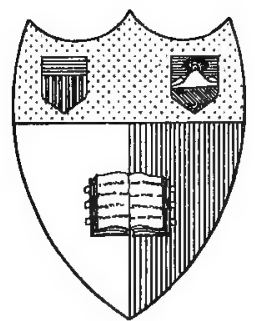

Aveu 盼ack

State Callege of Agrinulture

At (Tornell Alnüuersity Jlthaca, A. 照.

\section{Tibrary}


QL 781.883 ${ }^{\text {Cornell University Library }}$

The development of certain instincts and

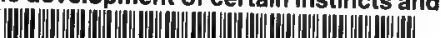




\section{Cornell University Library}

The original of this book is in the Cornell University Library.

There are no known copyright restrictions in the United States on the use of the text. 


\title{
BEHAVIOR MONOGRAPHS
}

Volume 1, Number 1, 1911

Serial Number 1

Edited by JOHN B. WATSON

The Johns Hopkin. University

\section{The Development of Certain Instincts and Habits in Chicks}

\author{
FREDERICK S. BREED
}

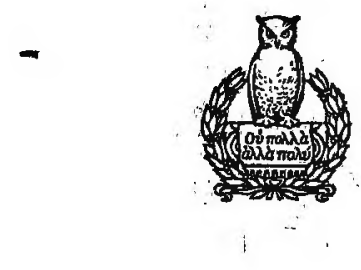

Published

at Cambridge, Boston, Mass.

HENRY HOLT \& COMPANY

34 West 33d Street, New York

G. E. STEChERT \& CO., London, Paris and Leipziq, Foreign Agenta 


\title{
The Journal of Animal Behavior
}

An organ for the publication of studies concerning the inetincts, habits and intelligence of organisms

The Journal contains a Department of Notes in which appear brief accounts of especially interesting and valuable observations of behavior.

Published bi-monthly at Cambridge, Boston, Mass., by Henry Holt and Company, New York. Each volume contains at least 450 pages with plates and text-figures.

The subscription price is $\$ 3.00$ per volume (foreign subscription $\$ 3.50)$ postpaid.

Manuscripts, subscriptions, and all business correspondence should be addressed to

THE JOURNAL OF ANIMAL BEHAVIOR

Emerson Hall, Cambridge, Massachusetts.

\section{Behavior Monographs}

\author{
For the publication of studies in behavior and intelligence which are \\ too lengthy or too expensive for acceptance \\ by The Journal of Animal Behavior
}

Published at, irregular intervals at Cambridge, Boston, Mass., in connection with the Journal of Animal Behavior, by Henry Holt and Company, New York. Each volume contains approximately 450 pages with plates and text-figures.

The subscription price is $\$ 3.00$ per volume (foreign subscription $\$ 3.50)$ postpaid.

Monographs may be purchased separately at prices varying with the cost of manufacture.

Manuscripts and inquiries concerning terms of publication should be addressed to the Editor of the Behavior Monographs,

JOHN B. WATSON,

The Johns Hopkins University,

Baltimore, Md.

Subscription to The Journal of Animal Behavior and the Behavior Monographs should be sent to Emerson Hall, Cambridge, Massachusetts. 


\title{
BEHAVIOR MONOGRAPHS
}

Volume 1, Number I, 1911

Serial Number 1

Edited by JOHN B. WATSON

The Johns Hopkins University

\section{The Development of Certain Instincts and Habits in Chicks}

\author{
FREDERICK S. BREED \\ Assistant Professor of Education \\ The University of Michigan
}

From the Harvard Psychological Laboratory

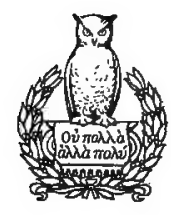

Published

at Cambridge Boston, Mass.

HENRY HOLT \& COMPANY

34 West 33d Street, New York

G. E. STECHERT \& CO., London, Paris and Leipzig, Foreign Agents

LL.

$M N$ 


$$
\begin{aligned}
& \text { Q4781 } \\
& \text { B83 } \\
& \text { Hy } 10221
\end{aligned}
$$




\section{CONTENTS}

INSTINCTIVE REACTIONS

I. Introduction and Statement of the Problem $\ldots \ldots \ldots \ldots \ldots \ldots$

II. Previous Experimental Work on Chicks............... I

III. General Apparatus........................... 4

IV. Observations and Experiments................

A. First Activities................... 5

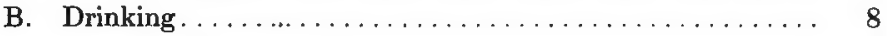

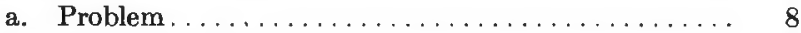

b. Method and tests................... 8

c. Discussion of results.................... 11

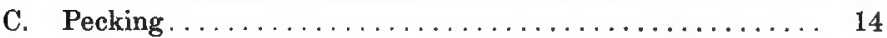

a. Apparatus and method ............... 14

b. Pecking artificially deferred............. 16

c. The natural development of the pecking reaction, with a collateral study of the effects of social influence. 21

1. Sources of error................... 21

2. Data and their significance............ 26

PART II. ACQUIRED REACTIONS ................... 41

I. Introduction and Statement of the Problem............. 41

II. Apparatus............................ 42

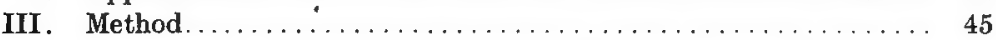

IV. Experiments................................ 48

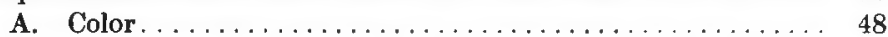

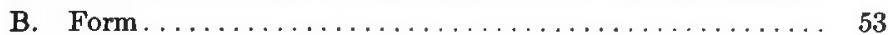

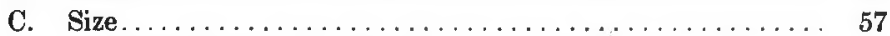

D. To What Extent Has Training General Value?....... 59

E. Some Effects of Modifications................. 67

F. The Persistence of Modifications..............., 71

Summary and Conclusions......................... 75 



\section{PART I. INSTINCTIVE REACTIONS}

\section{Introduction and Statement of the Problem}

The experimental work reported in this monograph was carried on during the years 1907,1908 , and 1909 in the Harvard Psychological Laboratory. The variety of chick used in all the work was the Barred Plymouth Rock. It is usually considered by poultry breeders a hardy chick, and for this reason more than any other was selected for these tests. The program of research began with a cursory study of the first activities of the animals, preliminary to a more detailed study of certain instinctive modes of response such as drinking, pecking, and "imitating." In the case of drinking, interest centered in the nature of the stimulus; in the case of pecking, in the accuracy of the reaction; as for imitation, the question was asked, In how far is social influence a means to improvement in the accuracy of pecking? After some knowledge of the natural tendencies of the chicks had been gained, an attempt was made to trace the course of development of certain habits of response to optical stimuli, as well as to study the interrelation and persistence of these habits. Quantitative methods were devised and applied wherever they suggested themselves to the experimenter.

The investigation was pursued under the immediate direction of Professor Robert M. Yerkes. What I owe to his searching criticism and fertile suggestions, a mere word of acknowledgment will not suffice to say. I shall be-only too glad if my immature effort reveal some trace of his admirable scientific spirit. I am also much indebted to my colleague, Professor Charles Scott Berry, for a critical reading of the manuscript, and to Dr. William F. Hauhart, Instructor in German, University of Michigan, for valuable assistance in reading the proof.

\section{Previous Experimental Work on Chicks}

Attention is limited in this résumé to the conclusions of investigators in regard to the accuracy of the pecking response. The results of some previous studies of the drinking reaction will be 
discussed in connection with the results of a study of the reaction reported later in the paper.

Spalding's ${ }^{1}$ observations on the pecking reaction have been quoted widely. Concerning the pecking of his chicks he says: "They did not attempt to seize things beyond their reach, as babies are said to grasp at the moon; and they may be said to have invariably hit the objects at which they struck-they never missed by more than a hair's breadth, and that too, when the specks at which they aimed were no bigger, and less visible, than the smallest dot of an $i$. To seize between the points of the mandibles at the very instant of striking seemed a more difficult operation. I have seen a chicken seize and swallow an insect at the first attempt; most frequently, however, they struck five or six times, lifting once or twice before they succeeded in swallowing their first food." (Italics mine.)

On the question of the accuracy of the pecking of chicks, Preyer 2 disagrees with Spalding. "I cannot admit," he says, "the supposed infallibility to within a hair's breadth. They miss in pecking by as much as two millimeters, though seldom. On the other hand, the attempts at swallowing frequently fail. Here it should be considered that even grown fowls are not sure in their pecking, seizing, and swallowing, as any one that observes closely may easily perceive. The accuracy is, however, marvelous at the very beginning." (Italics mine.)

Romanes, ${ }^{3}$ though reporting no experimental work of his own, deserves notice on account of his wide influence. $\mathrm{He}$ quotes Spalding extensively and with approval. For Romanes, perfection as applied to instinct means perfect adaptation independent of individual experience. He illustrates such perfection "by considering the wonderful accuracy of many among the highly refined and complex adjustments which are manifested by the newly-born young of the higher animals," (italics mine), citing first in his list of examples of perfection the pecking reaction of chicks as reported by Spalding.

Eimer ${ }^{4}$ experimented on chicks during two different years. The impression he got of the accuracy of pecking appears in the

\footnotetext{
${ }^{1}$ Spalding, D. A.: Instinct. With òriginal observations on young animals. Macmillan's Mag., 1873, vol. 27, p. 284.

2 Preyer, W.: The senses and the will. (1881.) Tr., New York, 1890, p. 67.

${ }^{3}$ Romanes, G. J.: Mental evolution in animals. New York, 1884, p. 161.

4 Eimer, G. H. T.: Organic evolution. Tr., London, 1890, p. 246.
} 
following report of the reactions of one of his animals: "At the first attempt it touched a grain with as much certainty as if it had pecked at millet for ever so long. But it did not succeed in taking up the grain in its beak. A second attempt immediately afterwards likewise failed, but at the third, which succeeded without a pause, the grain was grasped and swallowed, and now the little creature went on feeding as though it had done so for years!" (Italics mine.)

Mills ' thinks that some of Spalding's statements exaggerate. "Instinct is not the hard and fast thing it is sometimes supposed to be." On the basis of his own experiments he concludes: "Thus one (chick) may strike a crumb accurately every time it pecks, and pick it up on the first attempt; another misses, or shows great difficulty in getting it into the mouth." (Italics mine.)

Morgan ${ }^{2}$ concludes that the complicated process of striking, seizing, and swallowing "is performed so soon, and with so few trials - often at the third or fourth attempt - that one must regard the whole as essentially congenital in its definiteness, and look upon the few preparatory efforts as merely the steadying of the inherited organic apparatus to its work." (Italics mine.) By congenital response is meant one that is not the result of acquired skill.

Writing earlier on the same topic, Morgan ${ }^{3}$ had said the chicks "required a little intelligence, acting by and through experience, to perfect their (instinctive) activities. The instincts were very nearly, but not quite, perfect."

Thorndike " criticises Morgan's conclusions on the improvement in accuracy of the pecking reaction. "Lloyd Morgan, for instance, has chosen a dubious example of perfecting through habit in the seizing of bits of food by chicks. They often do fail to seize in their first experiences, as he observed, but they often, perhaps just as often, fail even after long experience." (Italics mine.)

Finally, a further matter, aside from the question of the

${ }^{1}$ Mills, W.: The psychic development of young animals and its physical correlation. Trans. R. S. Can., Sec. IV, 1895; or, The nature and development of animal 1 intelligence, New York, 1898, p. 262.

2 Morgan, C. L.: Habit and instinct. London and New York, 1896, p. 37.

${ }^{3}$ Morgan, C. L.: Introduction to comparative psychology. London, 1894, p. 207.

${ }^{4}$ Thorndike, E. L.: The instinctive reaction of young chicks. Psych. Rev., VI, 1899, p. 282 . Also, Instinct. Biol. Lectures, Woods Hole, 1899, p. 61. 
accuracy of pecking. Katz and Révész ${ }^{1}$ support Hess ${ }^{2}$ in the view that the hungry chick does not peck at food which it does not see. The former assert, for example, that a chicken in the dark-room, even if placed directly in the food, will not peck at it. The point happens to be vital to their method in some important work.

It has seemed best to report the essential conclusions of the writers mentioned, so far as possible, in their own words. It may not be amiss, however, to note two general impressions one gets from these studies of the pecking instinct: ( $\mathrm{I}$ ) that the reaction has a high degree of congenital accuracy, and (2) that it has less of this than was formerly supposed.

\section{General Apparatus}

The room used for these experiments had a western exposure. It was equipped with an incubator ${ }^{3}$ and a brooder " so that chicks for the experiments might be hatched at any time during the year in the laboratory. Such an arrangement also provided a good opportunity for complete knowledge of the history of the animals. For the sake of safety, economy, and convenience, both brooder and incubator were heated with gas. In addition to the regular thermostat on the incubator, a gas regulator : was attached to insure a more constant temperature. No trouble was experienced at any time in securing eggs of the special kind desired. The incubator and brooder were easily operated after a careful study of the directions that came with the machines. The percentage of fertile eggs hatched during the first year was 65.6. At times two settings were run simultaneously in the same incubator.

' Katz, D. und Révész, G.: Experimentell-psychologische Untersuchungen mit Hühnern. Zeitschrift f. Psych. u. Physiol. d. Sinnesorgane, 1909, Bd. 50, S. 93.

${ }_{2}^{2}$ Hess, C.: Ueber Dunkeladaptation und Sehpurpur bei Hühnern und Tauben. Archiv f. Augenheilkunde, 1907, Bd. 57, S. 298-316.

: Untersuchungen über Lichtsinn und Farbensinn der Tagvögel. Ibid., 1907, Bd. 57, S. 317-327.

Untersuchungen über die Ausdehnung des pupillomotorisch wirksamen Bezirkes der Netzhaut und über die pupillomotorischen Aufnahmeorgane. Ibid., 1908, Bd. 58, S. 182. von Tag-und von Nachtvögeln. Ibid., 1908, Bd. 59, S. 143-167.

${ }_{3}^{3}$ Junior No. 2, sold by the Prairie State Incubator Co., Homer City, Pa.

4 Indoor Brooder, No. 5, sold by the same firm.

- Single Float Standard Automatic Gas Regulator, secured from the Gas Consumers' Association, 18 Boylston St., Boston, Mass. 
Greater freedom of movement was provided for the birds by the use of two cages that were connected with the brooder. For litter, white sand and shredded alfalfa were found satisfactory. Sand is more suitable than ordinary earth where it is desirable to avoid dust. A chick food ${ }^{1}$ that is regularly on the market served our purposes very well. With the above simple equipment the chicks for the experiments were hatched and kept in the animal wing of the laboratory.

\section{Observations and Experiments}

\section{A. First Activities}

If one would have the ontogenetic history of a chick, he must begin his study of the development of activity while the chick is still within the egg. A movement of the egg tray was sometimes found to be the occasion of a chirp within the egg hours before the shell was chipped. Or, chirping at times occurred so loud that it could be heard distinctly in the room in which the incubator was situated, when the incubator was tightly closed and no external stimuli, such as noises and jars, were noticed. Before the shell was broken at any point, a continuous tapping sound could often be heard within the egg, somewhat as if the chick were pecking against the inside of the shell. The movements of chicks just after the shell was chipped were observed through the glass door of the incubator and also seen to good advantage when the eggs were removed from the incubator and put in an appropriate receptacle under an electric light. Upon these latter occasions the ragged edges of the egg shell near the beak of the chicken were removed to give a better view of the animal's movements. Upon the observations following the transfer of a chipped egg to a nest warmed by an electric light, I find the following note: "I broke away much of the shell. After bill of chick was entirely out of reach of the shell, the chick continued to make a sound somewhat like pecking, and that, too, without opening and closing the bill. The 'clicking' sounds seemed to accompany the rhythmical heaving of the animal, which I took to be breathing.

Eyes were open, apparently, as soon as chick had unfolded itself." At the next hatch another chick was observed under an electric

${ }^{1}$ Cyphers Chick Food, sold by the Cyphers Incubator Co., Boston, Mass. 
light in the same way. I follow my notes: "Made at times a regular clicking noise with breathing. Frequently clapped mandibles together.

Repeatedly raised bill with a lifting motion of the head. This reaction might bring tip of bill, and hence the bill scale, in contact with the shell. Opened mouth and lifted bill. Eyes closed while in egg. Sometimes pushed bill forward, following this movement with a clapping together of the mandibles."

While the chicks were still in the egg the legs were folded well up on the breast and the head was turned down toward the breast. After the egg was chipped so that the behavior of the animal could be observed, the lifting movement of head and beak above referred to was more frequent than any other. One much less often saw anything like a forward thrust of the bill.

The chicks came out of the eggs wet and, in a temperature of $103^{\circ} \mathrm{F}$., remained several hours before drying off. Spells of vigorous activity alternated with periods of passivity during the chicks' struggles just prior to hatching, as well as for some hours after hatching. The post-embryonic life of the chick in its early hours seemingly prolonged without interruption the life in the embryo. Chicks appeared to break the shell in two by a lifting, struggling movement of the head accompanied by a stretching, straightening movement of the legs. At least I have observed this combination of movements at the moment of hatching. Now if one watch carefully the behavior of a chick for some time after exclusion, he finds that there are these similarities between the later life within the shell and the earlier life outside the shell. The positions of the head and legs within the shell correspond to the positions assumed by these members during the passive states shortly after hatching. In both cases activity and passivity alternate, the periodicity of which alternation seems to be largely determined by intra-organic stimuli. If it be true that the legs participate in the action that finally breaks the shell, as I think they do, the lifting of the head and the pushing with the legs would be represented in the early post-embryonic life by those pulses of activity in which the chick lifts its head, rises to its feet, staggers a few steps, or struggles a few moments, and lapses again into the passive state. And just as the chick in the shell often claps its mandibles 
together without marked activity in other regions of the organism, so the chick during the first few hours after exclusion may be seen to work its mandibles in a similar way without disturbing its sitting posture. The pulses of activity, embryonic and post-embryonic alike, are often accompanied by loud chirping.

One might expect to find more unmistakable evidence of a pecking reaction while the chick is yet within the egg. There is an impression abroad that the chick pecks its way out of the shell. When the position of the head in the embryo is taken into consideration, one can see two reasons why most of the movements of the head are lifting movements, and not pecking reactions: ( $\mathrm{I}$ ) the lifting movement tends to free the head and neck from their folded position, and (2) pecking would seem to be a difficult matter with the head folded down on the breast in this wise. Besides, the lifting reaction does actually break shell and tear confining membranes, and so is effective in releasing the chick. It may be found that the chick does not peck its way out of the shell. However, the matter will need more careful study. For hours I have watched chicks laboring in the egg to discover a clear case of the pecking reaction. At times, before the egg has been broken in two, one does see short, quick, forward thrusts of the bill followed by working of the mandibles. And chicks only a few hours out of the egg may be observed repeatedly executing what might be called a pecking reaction " into the air," followed by clapping together of the mandibles. The following note describes the reaction more adequately: "Chicks after exclusion, on becoming aroused from a dormant period, often open bill with a chewing motion and sometimes thrust the bill forward sharply into the air even without fully opening the eyes, no object, apparently, being pecked at. Noticed a number of times."

The following activities, generally recognized as instinctive, were observed within the incubator on the first day, before the chicks were completely dry: Preening the down of the neck, wings, and breast; flapping of wings; chirping; walking; pecking; lying on side and stretching out legs (in the rays of a I6 c.p. electric lamp); following a moving object with a motion of the head; and chirring. Scratching, twittering, and wiping of the bill have been noticed on the second day when the chicks ) were taken out of the incubator for the first time and placed on 
a black cardboard for their pecking tests. Our knowledge of the time of appearance of the various instincts should not be left to depend entirely upon chance stimuli. Who shall say that a given reaction might not have occurred much earlier if the appropriate stimulus had been provided?

The chicks, while still in the incubator, are known to be positively phototropic. The incubator trays that have near the glass door a trap through which the chicks fall to a screen below, depend for their effectiveness on the fact that the chicks crowd toward the light.

B. Drinking

a. Problem.-In the study of instincts from the objective point of view, interest naturally centers first in function, second in structure. The activities which are known as instinctive must be analyzed into component units of behavior, of which they are nearly always complexes. Furthermore, no account of instinct will be satisfactory, no explanation complete, until re understand the structure of the machinery involved in each action. But so much accomplished, this is not all. These structures are not of such a nature that they in some way get themselves into action. So far as we know, they have no inherent principle of spontancity. Intra- or extra-organic stimuli are necessary to touch them off. Environment in the form of energies external to the structures and additional to the functions seems to be a sine qua non. Hence a complete understanding of instinctive actions will include a detailed knowledge of the "objects" in conjunction with which the particular activities manifest themselves. In the following bit of work on the instinct of drinking, consideration of the problems of function and structure is made secondary to an inquiry into the nature of the extra-organic stimulus.

b. Method and Tests.-Chicks no. 69 to no. 8r, inclusive, were hatched during the afternoon and evening of Dec. 2 and the morning of Dec: 3, 1908. Beginning with Dec. 3, their pecking had been tested in the regular manner each day. As the animals finished the first pecking tests they were marked, numbered, and transferred from the incubator to the brooder. Although given food and freedom to run about in the litter, they were allowed nothing at all to drink until tested as described below, neither were they permitted to see other chicks drink. A pos- 
sible chance of coming in contact with something like water was afforded through deposits of waterish excrement. Even if this as a stimulus were able to elicit the drinking reaction, the fact that the chicks in the incubator rested on an elevated wire screen lessened the probability of the occurrence of such a stimulus. In the brooder the floor was constantly covered with the regular litter from one to two inches deep. On Dec. 5 , from 3 to $5: 30 \mathrm{p}$. m., when the chicks varied from 2.5 to 3 days of age, they were brought one at a time to the experiment table, just as if they were to be given a pecking test, and were returned to the brooder each as its drinking test was completed. In front of each animal was set a clean watch glass containing fresh water devoid at the start of bubbles or sediment. The watch glass rested on a square piece of smooth, white, plain note paper. As the observations on each chick were completed, the soiled paper was replaced by a clean piece, the dish was washed, the water renewed, and bits of food or drops of water carefully brushed from the table. The report of the observations follows in the order in which the chicks were tested.

While no. 70 was eating on the table, the watch glass, in the manner set forth above, was presented. The chick pecked grains which had been scattered over the white paper and then ran its bill in a forward direction along the paper in the drinking reaction. This it did repeatedly. Later I helped it to find the water in the dish. It seems unnecessary to state that precaution was taken to see that the paper was dry.

No. 72 was tried next. It pecked the plain, clean, white paper. Before the water was found by no. 72 , no. 70 was brought out and allowed to drink in the presence of no. 72 . No. 72 followed no. 70 about, performing the drinking reaction along the edge of the dish. One of the chicks stepped into the dish and carried some water to the paper surrounding it. No. 72 got its bill into this and forthwith responded with the drinking reaction. Then it wandered about the table and ran its bill along the black leather of my watch fob, $30 \mathrm{~cm}$. distant from the dish, giving the drinking reaction. This does not mean that it touched the fob with its bill and then lifted its head in the manner so well known. The reaction to the fob was just what one sees when he watches a chick gathering water into its bill and throat while the bill is inserted in the liquid. This reaction is 
not easily confused with pecking. It was repeated on the silver charm of the fob.

No. 7 I learned to drink by pecking at a bit of excrement that chanced to get into the water. Time after time it pecked about the edge of the dish. It was not observed to dip its bill into clear water. It secured water first by pecking and persisted in getting it this way throughout these first trials.

No. 74, when set on the table, pecked the edge of the white paper. First contact of the bill with water came from pecking a drop of water deposited on the paper. I put its bill into the water in the dish, whereupon it reacted to the situation by pecking the edge of the dish.

No. 77. By catching the paper on which the dish rested, the water was caused to wave slightly in the presence of the chick. Its head went down hard in a pecking reaction and hit the bottom of the dish, following which the head was lifted in the manner characteristic of drinking. On the second trial the head approached the water not in the manner of pecking but of drinking. The pecking approach to an object is decidedly different from the drinking approach. In contrast to the sharp descent upon the object in the former, the low gentle reaching movement in drinking, accompanied by a straightening of the neck, opening of the mouth, and a peculiar motion of the throat, is most marked.

No. 69. The surface of the water was agitated as for no. 77. No. 69 pecked directly into the water. Shortly afterward it pecked the water without my shaking it and at a point where I could discern no special stimulus like a bubble or particle of food. The positions of chick and dish were such that light reflected from the water might have been a factor.

No. 75. Pecked edge of the white paper. Pecked about the edge of the dish and its bill slipped into the water. The chick then pecked the edge of the dish twelve times, the bill getting into the water during some of these reactions. Pecked twice into the dish and got a little water. Of the next twenty reactions all were pecks and all but two were directed toward the side and edge of the dish.

No. 8o. I thought I observed the chick performing the drinking reaction on a piece of glazed kymograph record paper about $3 \mathrm{~cm}$. square that I vibrated between two fingers in front of 
the chick. But the reaction came out unmistakably a moment later on the side of the glass dish. The water was still, the glass was dry, and the chick ran its bill along the edge of the glass exhibiting plainly the drinking movements without touching the water. It later found the water and began to drink without assistance.

No. 8I. On the first attempt it dipped its bill into the still water and drank from a point at which I could distinguish no special object in the water. While crouching by the dish drinking, as its bill was coming down slowly at the termination of a drinking reaction, it turned its head to the left, touched its bill gently to a bit of dry chick food lying within reach, and performed the drinking reaction upon it. It did not eat the grain.

No. 79. Pecked side of dish. Ran beak along edge of dish. Its beak (accidentally) slipped into the water. After this the chick began to drink. Frequently ran beak down the outside of the dish.

No. 73. Did not peck at dish nor find water. No. 74 was placed on the table with no. 73. No. 74 pecked five times at the edge of the dish, not touching the water, when no. 73 began to peck near the same place. The latter's bill slipped into the water and it began to drink energetically.

No. 76. I shook the water in its presence and it pecked into it. It began to drink and twitter. Pecked the edge of the dish. Ran its bill along the outside and inner edge of it.

c. Discussion of results.-Spalding, ${ }^{1}$ commenting on the drinking of chicks, remarks: "It also appeared that, though thirsty, they did not recognize water by sight, and they had to some extent to learn to drink." Discussing the same instinct, Morgan " says: "The statement of fact (so far as my observations go) that I made was this: That the sight of still water evoked no instinctive response; but that the touch of water in the bill at once evoked the characteristic instinctive behavior." Mills ${ }^{3}$ expresses his opinion thus: "It is not primarily so much the sight, but rather the touch of water that in the very first instance leads to drinking."

${ }_{1}$ Spalding, D. A.: Loc. cit., p. 288.

${ }^{2}$ Morgan, C. L.: The habit of drinking in young birds. Science, N. S., 1896, vol. 3 , p. 900 .

${ }^{3}$ Mills, W.: The nature and development of animal intelligence. New York, 1898, p. 281 . 
On the basis of these and similar observations, it has been asserted that a chick swallows water instinctively, but must learn to drink by imitation or accident; that is to say, the drinking instinct requires supplementation. A passage from Baldwin " will illustrate: "In the case of the fowl's drinking, it is not the mere fact that drinking and eating may differ in the degree to which the performance is congenital; the reports seem to show that this varies in different fowl; but that instincts (in this case drinking) may be only half congenital, and may have to be supplemented by imitation, accident, intelligence, instruction, etc., in order to act, even when the actions are so necessary to life that the creature would certainly die if the function were not performed. That is the interesting point."

For the sake of clearness in the discussion of drinking, the parts of the drinking complex must be more sharply distinguished. There is (I) the approach to the object, elicited evidently by optical stimulation, (2) a sort of rhythmic movement of mandibles and throat, which brings the object within the mouth, and (3) the swallowing activity, which is evoked by stimuli resulting from the contact of water with the mouth, and which is marked by an elevating movement of the head and neck. We find an exactly parallel series upon analysis of the pecking reaction: (I) striking, (2) seizing, and (3) swallowing. Any full account of the drinking instinct must include the approach to the object as well as the subsequent manipulation.

No one, I think, will deny that the touch of water in the bill evokes reaction 3 of the above series. And we know that chicks may get this appropriate contact-stimulus indirectly by pecking. Furthermore, the drinking of one chick in the presence of another often stimulates this other to become active about the water and thereby leads to its drinking. That is, drinking usually does begin as the result of a contact stimulation mediated by the prior activity of the pecking and imitating instincts. But this at once suggests the further question, Are imitating and feeding necessary precursors to reaction $I$ of the series, the movement of approach? The results of the foregoing experiments seem to show clearly that the drinking instinct is selfdependent in so far as its relation to these other instincts is

${ }^{1}$ Baldwin, J. M.: Instinct. Science, N. S., 1896, vol. 3, p. 669. 
concerned. When chicks, without having previously drunk, respond with the drinking reaction to the surface of smooth white note paper, the edge of white glazed kymograph paper, or the edge of a glass dish, all these objects must be supposed to have some quality or qualities, undoubtedly visual, which evoke the drinking reaction. Here are stimuli that call forth a first drinking reaction independently of other instincts. If further evidence were needed to substantiate the conclusion that the instinct is self-dependent, it is found in certain other observations. After the first actual drinking, the drinking response was made to a grain of food, a piece of black leather, a silver ornament, and, in the case of chicks other than those studied in the special experiment, to a line in some dust on a smooth surface, a white spot on the experiment table, the clean surface of black cardboard, and the polished surface of a table.

Thus the feeding and drinking instincts are more similar than writers have hitherto supposed. In the case of feeding, the fact seems to be that newly hatched chicks respond to a great variety of objects indifferently, and only later come to select those which are food from the rest; in the case of drinking, the observations show that, if the need be sufficiently urgent, a large variety of objects in like manner elicit the action, and apparently with a like result. The indiscriminate use of reaction $\mathrm{I}$ of the drinking instinct may bring the appropriate stimulus for reaction 3 , without the co-operation of the pecking and imitative activities. The drinking instinct, therefore, does not "have to be supplemented by imitation, accident, intelligence, instruction, etc., in order to act."

Finally, is still water a sufficient stimulus for the act of drinking? I am by no means ready to say it is not. I regret that my experiments are not so complete on this point as they might have been. There is general agreement at present that chicks do not begin to drink in response to this optical stimulus. But deprive them of water for a sufficient length of time after hatching and perhaps the "sight of still water " will evoke this instinctive reaction. It is not improbable that the effective element or elements in the objects which have been observed to draw forth the reaction are common also to water. 


\section{Pecking}

a. Apparatus and method.-A detailed and prolonged study of the pecking reaction was now attempted. The literature on this topic reveals the fact that the interest in the accuracy of this instinctive activity has been the central one. As suggested before, the accuracy of the reaction became the central interest also in the investigation that is reported in the following pages.

The apparatus used in these experiments was very simple. A table with a hard polished surface was set near a window where there was good light. To this table the chicks were each morning brought, one at a time, and permitted to eat in a natural way from the surface of a piece of black cardboard about 20 $\mathrm{cm}$. wide and $25 \mathrm{~cm}$. long. Carried daily to and from the experiment table, the chicks became so habituated to the transfer that the fear response did not enter in to mar the value of the results. From the first the chicks ate from my hand, and soon many of them energetically followed the hand from point to point, gathering up the bits of food as they were dropped. A little later many even gave the "food twitter" while in my hand on the way from the brooder to the table. But it may not be said with accuracy that the chicks became "habituated " to the operation, if at any age without previous trials they submitted, without signs of being disturbed, to the conditions of the experiment. As a fact, they did not thus submit. Animals that had not been used in the pecking tests nor been handled previously in any other experiment were brought to the table for a control test. They usually struggled when picked up and seemed so disturbed by the situation, when set on the table, that they would not eat at all. This was especially true if they had been allowed to live in the brooder unmolested for two or three weeks.

For the first tests, which were conducted on the second day, because of the physical weakness of the chicks and their indisposition to eat on the first day, slightly moistened bread was used, of such a consistency that it could be rolled between the fingers into food particles of suitable size. After the second day Cyphers Chick Food, slightly moist, replaced the bread pellets in the tests. This, which was the regular food of the chicks, is a mixture of whole wheat, Kaffir corn, cracked corn, millet, etc. 
The food dish was kept out of the chicks' field of view and the bits of food to be pecked at were dropped by hand upon the cardboard in such a way that a particle would not be in motion when a chick pecked at it. From one to three grains only were dropped at a time. This proved advisable because the number of reactions elicited by more than three grains, considering also the possible variety and rapidity of the reactions, made it difficult at times to secure an accurate record.

In a letter published by Mills, ${ }^{1}$ Bumpus ${ }^{2}$ has suggested that the different aspects of the pecking reaction be distinguished. For these separate parts he proposed the terms seizure, mouthing or mulling, and deglutition. The terms used by Morgan ${ }^{3}$ seem much more appropriate-striking, seizing, and swallowing. It is interesting to. note that Spalding ${ }^{4}$ distinguished these phases of the reaction and employed the same terms. These we have adopted, using in addition the term missing for failure to hit the object. As they appear in our records, these terms have the following definite meanings: (I) Missing denotes all cases of the pecking reaction in which the bill fails to hit the particular object supplied by the experimenter; (2) striking, those cases in which the bill hits the object without seizing it; (3) seizing, cases in which the object is grasped momentarily in the bill and then dropped; and (4) swallowing denotes what may be termed the perfect or complete reaction, the object being struck, seized, and swallowed in an errorless series or chain of movements. To facilitate the taking of records, the numerals $\mathbf{I}, 2,3$, and 4 were used to represent missed, struck, seized, and swallowed, respectively. Note was taken, of course, of the reactions independently of the number of food particles pecked at, for a single grain might call forth a half dozen reactions in succession. For example, suppose one millet seed brought the result $\mathrm{x}-2-3-4$. In this case the chick first missed the grain, on the second reaction it struck it but did not get hold of it between its mandibles, on the third attempt it caught it in its bill but dropped it, and on the fourth, it struck, seized, and swallowed the grain without error.

${ }^{1}$ Mills, W.: The nature and development of animal intelligence. New York, 1898 , p. 296.

${ }^{2}$ Bumpus, H. C.: Instinct and education in birds. Science, N. S., 1896, vol. 4, p. 213.

Morgan, C. L.: Habit and instinct. London and New York, 1896, p. 37

${ }^{4}$ Spalding, D. A.: Loc. eit. 
For this, as well as for all other work, the chicks were marked by colored yarns tied on their legs. Each animal thus identified was assigned a number by which it was afterwards known and referred to.

b. Pecking artificially deferred.-The experiments of Spalding, ${ }^{1}$ in which chicks on leaving the shell were blindfolded with little hoods or kept in a flannel bag, have attracted much attention from students of instinct. Spalding sought to ascertain the facts in regard to instinct. In his time the skeptical were holding "that all the supposed examples of instinct may be-for anything that has yet been observed to the contrary-nothing more than cases of rapid learning, imitation, or instruction." The hooding device was intended to permit the chicks to acquire " enough control over their muscles to enable them to give evidence as to their instinctive power." In other words, the aim was to test the activity of pecking when the factor of acquisition was eliminated. The conclusions of Spalding as to the accuracy of pecking, previously referred to, are based on the results of these tests.

In the similar tests that are reported below, the purpose was to measure the accuracy of the pecking response under circumstances like the above. Not much success was achieved by hooding the chicks. Other means of excluding the light were relied upon. After being tested the chicks were placed in the brooder with the rest of the flock.

On Dec. IO, I907, chick no. 8, immediately upon hatching at $\mathrm{I}: 3 \circ \mathrm{a}$. m., was blindfolded and transferred to the brooder where it was kept until ro a. m., Dec. 12, under the curtained hover in a black-lined box at a temperature of $103^{\circ} \mathrm{F}$. This box, open at the top, was enclosed in a green flannel bag. At ro a. m., Dec. I2, the chick was brought carefully to the experiment table for a pecking test. Almost immediately it twice pecked the board upon which it stood. The particular stimulus to the reaction was not apparent. Then followed, in a series, seven reactions, in all of which the chick missed the pellet of bread (rolled as previously described) which elicited them. Seven more pecks at the same crumb followed without hitting it. Then the board on which it stood drew forth two more reactions, when the pecking was interrupted by an awk-

${ }^{1}$ Spalding, D. A.. Loc. cit., p. 282. 
ward attempt to bite its wing. After a spell of violent chirping, it missed twice, and hit as often, a spot on the board upon which it stood, following which it lifted a foot to scratch its head and lost its balance. Then four pecks at a crumb missed, but a fifth was successful and the crumb was swallowed-the first perfect reaction. Thereupon came a series of 79 pecking reactions in no one of which a particle of food was hit, seized, and swallowed in a chain reflex. In many of these reactions the head wobbled from side to side as the bill moved slowly toward the object pecked at. On the twentieth reaction in this series of 79 , the object was seized in the bill and apparently rejected by the chick. Prior to this, excluding the one perfect reaction, bits of food had been seized on two occasions but dropped through seeming lack of skill. Just preceding an interval occupied in preening the feathers of its wings, an interval which marked the end of the 79-reactions series, there occurred a group of five reactions, in three of which the object was struck and in the other two seized. It is but fair to state that many of the pecking reactions were not in the direction of food particles, there being something about the bare surface upon which the chick stood that drew the reactions forth. In the summary of the chick's record these reactions have been classified with the I's. At this point it was incidentally noticed that the chick followed with both head and eyes a movement of the experimenter's hand. After the above mentioned preening diversion, twelve more pecking reactions ensued: seven I's, one 2 , and four 3 's. Then the animal hesitated long enough to scratch its bill with its foot. The next nine reactions, of which three were I's, five 2 's, and one a 3 , came out in straggling order, interrupted by gaping, pecking of toes, scratching the bill, and preening the feathers of the breast.

When tested by a moving object, a moist bit of bread swinging by a black silk thread, the chick's first impulse was manifestly toward it, but later it acted as if afraid. During these tests the chick appeared in no danger, except by accident, of stepping off the edge of the piece of cardboard upon which it was placed, when the cardboard was so arranged that the outer edge of it coincided with an edge of the experiment table. The animal could easily be pushed away from the edge but, when near the edge, resisted strongly if pushed toward it. This same 
behavior was noticed in other chicks. For example, no. I4, the day after it was hatched, when on the experiment table for the first time, was pushed toward the edge. It resisted by bracing its legs in front of itself and hurried back from the edge as soon as it was released.

The summarized record of no. 8 is as follows:-

$$
\begin{aligned}
& I-I-I-I-I-I-I-I-I-I-I-I-I-I-I-I-I-I-I-I-2-2-I-I-I-I-4-I-I \\
& 2-I-I-I-I-I \quad I-I-I-2-2-3-I-2-I-I-3-3-I-I-I-I-I-I-I-I-I-I- \\
& I-I-I-I-I-I-I-I-I-I-I-I-I-I-I-I-I-2-2-I-I-I-I-I-I-I-I-I- \\
& I-I-I-I-I-I-I-I-I-I-2-I-I-I-2-2-2-3-2-2-3-I-I-3-I-I-I-I-I \\
& -2-3-3-3-I-2-2-2-I-2-2-I-3 .
\end{aligned}
$$

Attention has been called to the fact that the chick pecked the cardboard at a place where no particular spot or grain was discerned by the experimenter. Not only that, but, like a child repeating da-da, it sometimes continued with a rapid succession of reactions at or near the same place. Twenty-five of the long series of I's, immediately following the third 3 in the record above, represent such pecks at the bare card. They came out in three groups, first 2 , then 18 , and after an interval the other 5. To see a chick hammering in this way $r 8$ times in rapid succession, and hard, too, at apparently " nothing," reminds one of the mere exercise of a function, and suggests what Baldwin has termed the circular reaction.

A comparison, in regard to accuracy, of no. 8's record, with the numerous records which will be presented later, will show that this chick was below the arerage efficiency in its first test. It is not unusual to find normally kept chicks that do not make a single swallowing reaction in the first twenty trials on their second day, and chicks sometimes fail at this age to seize a single grain in the same number of trials, but I have found only one in the regular tests that failed to strike at least one grain in the first twenty reactions. There were instances in these first tests in which the side of the chick's bill touched the side of the object as the bill slowly passed and hit the cardboard. These were recorded as I's, here and elsewhere.

Naturally, after being kept 56.5 hours prior to the tests under the conditions previously described, the chick's down was ruffled and it lacked the general sleek appearance of its unconfined mates. Whether it was deficient in anything else but the results 
of practice, I cannot say. The confinement did not do it any permanent injury, it seems, for it continued in the pecking tests, lived to make a perfect record after an average number of trials in later black-blue color selection tests, and survived after that till it was killed and dissected for sex determination on March I4, Ig08. Its record in the later pecking tests is submitted in table $I$. This table shows the accuracy of its pecking during the nine days after Dec. I2, on the basis of twenty reactions a day.

TABLE 1

Chick no. 8. Dec. 13-21, 1907. Pecking

\begin{tabular}{|c|c|c|c|c|c|c|c|c|}
\hline Date................. & 13 & 14 & 16 & 17 & 18 & 19 & 20 & 21 \\
\hline Missed. & 2 & 1 & & & 2 & & & \\
\hline Struck. & 3 & 4 & 7 & & 2 & 2 & 2 & \\
\hline Seized . . . & 1 & $\overrightarrow{1}$ & & 3 & 1 & & & 1 \\
\hline$\ldots \ldots \ldots \ldots$ & 14 & 14 & 13 & 17 & 15 & 18 & 18 & 18 \\
\hline
\end{tabular}

The record of this chick in its first test is not presented as typical, but is submitted to show how a chick may act under conditions like those set forth above.

Two other chicks, no. 7 and no. I4, of the same brood as no. 8 , were enclosed at the same time in the dark-box with no. 8 . These chicks were helped out of their shells just as they were hatching and transferred at once to the dark-box. They were not hooded, but even if they had been securely' blindfolded, little would have been gained, for the light that reached the interior of the black-lined box was negligible in amount.

No. 7 was brought to the light for its tests at $\mathrm{x}: 05 \mathrm{a} . \mathrm{m}$., Dec. II, after 33 hours, $35 \mathrm{~min}$. in the dark-box. My notes on its behavior are as follows: "Followed moving object (hand) with head. Pecked wood over edge of black cardboard. Wiped bill on board. Pecked three times at a pellet of bread moistened with milk. Missed it each time. Chick pushed bill slowly toward bread crumb on the black surface-a slow peck. Missed crumb, weak peck. Bit toes. Touched small white speck with bill. (The experimenter did not point at objects.) Rested under hand with its eyes closed. Missed a crumb twice. Missed another. Moved bill toward it slowly. Chick able to stand. Pecked at feet and lost balance. Bit wing twice. Pecked black cardboard. . Chick seemed cold and was returned to the brooder." The record of no. 7 's pecking at food particles and 
spots on the card runs thus in its first test, as detailed above: $\mathbf{I}-\mathbf{I}-\mathbf{I}-\mathbf{I}-\mathbf{2}-\mathbf{I}-\mathbf{I}-\mathbf{I}$. Table $\mathbf{2}$ shows the accuracy of its pecking from Dec. 13 to Dec. 26.

TABLE 2

Chick no. 7. Dec. 13-26, 1907. Pecking

\begin{tabular}{lrrrrrrrrrr} 
Date . . . . . & 13 & 14 & 16 & 17 & 18 & 19 & 20 & 21 & 24 & 26 \\
\hline Missed....... & & & & & 1 & & & & & \\
Struck. . . . . & & 7 & 5 & 2 & 3 & 1 & 7 & 1 & & 2 \\
Seized...... & 3 & & 3 & 1 & 2 & & & 1 & 1 & 1 \\
Swallowed. . & 17 & 13 & 12 & 17 & 14 & 19 & 13 & 18 & 19 & 17
\end{tabular}

Chick no. I4, as just stated, had been placed in the same enclosure with no. 7 at the same hour on the same date, and was kept there till 4:25 p. m., Dec. I. After $3^{8}$ hours and $55 \mathrm{~min}$. thus enclosed, it was tested in the same way as no. 7 . On the first pecking attempt it pushed its bill, mandibles closed, into a bread crumb. Then followed six pecks that missed. Attempted to scratch its head and lost its balance. Seemed weaker than the other chicks. Pecked toes and bare black cardboard; scratched bill. A second time it pushed its bill slowly into a bread crumb. Then followed a reaction in which it missed, etc. The summarized record for this test appears thus: 2-I-I-I-I$I-I-2-I-2-2-I-2-2-2-2-I-3-2-2-2-2-3-2-2-2$. The accuracy of its later pecking, Dec. I3 to Dec. 26 , is shown in the following table:

TABLE 3

Chick no. 14. Dec. 13-26, 1907. Pecking

\begin{tabular}{lrrrrrrrrrr} 
Date . . . . . & 13 & 14 & 16 & 17 & 18 & 19 & 20 & 21 & 24 & 26 \\
\hline Missed....... & & & & & 1 & & & & & 1 \\
Struck....... & 9 & 6 & 12 & 6 & 5 & & & 3 & 4 & 1 \\
Seized...... & 2 & 1 & & 1 & & & 1 & 1 & 2 & 18 \\
Swallowed. . & 9 & 13 & 8 & 13 & 14 & 20 & 19 & 16 & 14 & 18
\end{tabular}

A comparison of the records of these three chicks for Dec. I 3 with the corresponding records of five others of the same age which were not subjected to confinement in darkness shows that there was no noteworthy difference in the development at this time. The averages of the three chicks on the above date were $7,4,2$, and $\mathrm{I} 3.3$ for reactions $\mathrm{I}, 2,3$, and 4 , respectively, while the other five chicks averaged .8, $1.4,4$, and I 3.8 for the same reactions, in a total of twenty reactions. On the 
other hand, the development of no. 8 on Dec. I 2 was far behind that of any chick in the above group of five on the same date.

It looks very much as if (I) the development of the instinct was retarded by disuse, and (2) the retardation was quickly overcome with use. Several attempts to repeat these dark-box tests with other chicks failed. On these later occasions the plan was conceived of placing the dark-box inside the incubator just before the expiration of the period of incubation, depositing therein a few of the eggs, and allowing the prospective chicks to hatch and remain in the dark enclosure till needed. For some unforeseen reason the chicks did not appear.

We turn now to a more thorough investigation of the "congenital definiteness" of the pecking reaction, with a view to a quantitative determination of its course of development.

c. The natural development of the pecking reaction, with a collateral study of the effects of social influence. I. Sources of error.-In addition to what has already been said of the method of taking records, the important sources of error might be mentioned. Investigators have noted the fact that the chick, if it misses the grain at all, usually comes within a hair's breadth of it. Hence, when the animal is working rapidly, it is sometimes difficult to determine whether the bill hits the grain or not. In the work herewith reported, a chick was credited with reaction 2 if there was any doubt between I and 2. In other words a I was recorded only when the miss was positively observed. The fact that more missing reactions seem to be recorded among the later results may be due rather to modification of the experimenter than to variation in the groups of chicks. I feel quite confident that I became a more efficient observer of the missing reactions as the work progressed.

Again, there are times when reactions 2 and 3 may be easily confounded. A grain seized simultaneously with the strike may almost immediately fly from the bill-like a cherry pit squeezed between the fingers. This reaction, properly 3 , may be confused with the reaction where the grain is driven from its position by the mere impact of the bill. Still, as one becomes familiar with such cases, the difficulty of distinguishing them almost wholly vanishes.

Occasionally fewer than the regular number of reactions in the daily series are reported for a given date. In such cases 
the cause was usually the refusal of the chick to eat. This difficulty was soon obviated by carefully controlling the amount of food allowed the chicks, and compelling them to scratch for it in the litter. The objection also was thereby forestalled that many chicks under an improperly regulated food supply might react the required number of times but below their highest efficiency.

Not only did these difficulties threaten to affect the reliability of the results, but an additional reaction came in to complicate the record. When the food supply was improperly controlled, the chicks would quite often reject grains, that is, lift them from the ground with the bill and then either drop them or throw them some distance without attempt to recover. Forcible rejection is distinctly a reaction in addition to reaction 3 . It involves control of the grain by the mandibles-sufficient, one should suppose, for manipulation without error prior to swallowing. If this be true, it is manifestly unfair to charge the chick with a degree of imperfection by recording reaction 2. Yet, one is not at liberty to credit it with reaction 4 . A clear instance of rejection should simply not figure in the records. But this disturbing feature was practically eliminated when the chicks were confined in the brooder at night and were given the proper amount of food only after the morning tests. I say practically eliminated, because, even after taking the precaution mentioned, some chicks in time came to eat one kind of grain and not another. For example, I have found an occasional chick that discriminated in this fashion between millet seeds with the shell on and those without the shell, to say nothing of preferences for wheat as against corn, millet as against wheat, and the like. In such cases the chick was fed what it readily ate.

Another variation, which we may term switching of the bill, occasionally appeared. It is similar in character to the forciblerejecting movement, and was observed in some very young chicks. It is executed while the food particle is held firmly between the mandibles. It is mentioned here rather as an additional type of reaction than as a source of error, for it did not noticeably affect the records.

I have so many times observed chicks, especially very young ones, peck where I could see nothing but bare wood or cardboard, that I am compelled to believe that the stimulus to the 
pecking reaction need not be some object of a size convenient for eating. In line with this consideration is the further fact that the bill of the chick is not only a feeding but a testing organ. If anywhere, the "oral sense" has its organ here. The chick often digs in the litter with its bill when the reaction is distinctly not the pecking reaction. While trying to escape from the reaction box of a discrimination apparatus in a dark-room, chicks often approached a sheet of clean, smooth, lighted opal glass and pecked it. In the same box they pecked the black walls and especially the black cardboard closing the exit, when they happened to enter the wrong side and found their egress barred. But, except where specially noted, no missing reactions were recorded when no object to be pecked was supplied.

Besides the occasional variation in the missing reaction in which the animal pecked the bare cardboard when no other stimulus seemed to be present, there were two other reactions that were easily distinguishable from each other and from the regular missing reaction, but which were, nevertheless, put down in the records as reaction $\mathrm{I}$. These variations in the missing reaction were altogether so few in number, compared with the many hundreds of reactions recorded, that the averages are not materially affected by the classification of them with reaction $\mathrm{I}$. I refer here (I) to cases in which the chick pecked in the direction of the grain but did not reach it, and (2) to cases in which the bill struck the cardboard almost exactly midway between two grains. Of the first of these I have records of thirteen instances; of the second, thirty-six instances. Case one needs no special comment. The bill each time was going in the right direction, but the innervation seemed to be insufficient. Case two is, if anything, more interesting. When the typical instance of it occurred, the grains were usually about I cm. apart, of about equal size, and the line connecting the two ran about normal to the direction in which the chick was oriented, the chick standing directly before them. One could say with little certainty that this was an instance of pure missing. We have already agreed with other observers that the chick from the first rarely missed by more than a hair's breadth an object pecked at. But here was a case where a chick from one to four weeks old missed a grain by 4 to $6 \mathrm{~mm}$. Not only that, but I have five records where in a situation like that described above the chicks pecked twice 
in succession between the two grains, and one record in which there were four such successive pecks. There are two other observations that help to explain this reaction. On Dec. 23, no. 70 and on Dec. 29 , no. 87 , were working rapidly in their regular pecking tests. The pecking activity was interrupted momentarily while the bill poised in air above and about midway between two grains approximately $\mathrm{I} \mathrm{cm}$. apart. On Dec. 24 , no. 76 was on the experiment table going through its pecking test. In the presence of two grains lying as those in the preceding cases, its pecking reaction started in the direction of one of the grains and was completed by a perfect reaction upon the other. I concluded, therefore, that case two was not an ordinary instance of missing, but that the reaction in response to one of the stimuli exerted an inhibitory effect on a reaction in response to the other. The poising of the bill in air, the zigzagging first toward one grain and then toward the other during a continuous forward movement of the bill, and the striking of the cardboard fairly between the grains, have at bottom, I believe, the above common principle of explanation.

In view of the recent important work of Hess and that of Katz and Révész, I feel impelled to report some activities of the chicks in the dark-room. The assumption that hungry chicks do eat when they can see the food is ordinarily true, but the second assumption, that chicks do not eat when they cannot see it, surely is not borne out by observations of the behavior of chicks in the dark-room. During some studies of individually acquired reactions, the chicks were placed in a dark-basket in the dark-room for ten minutes prior to the regular daily tests. The basket which was used for this adaptation work was a willow waste-paper basket lined with black cloth and covered with the same material. Food was placed in the bottom of the basket before the basket and chicks were taken into the darkroom. The animals were thus allowed to eat in the basket a short time before the period of darkness adaptation began. Again and again, after the basket was taken into the dark-room, the door closed, all lights turned off, and the basket covered, the animals continued to eat the chick food in the bottom of the basket. I have also gone out of the dark-room and returned at the end of the ten minute period to find the chicks eating. The darkness was so nearly absolute in the room that I could not 
see the dark basket while sitting in a chair nearby. The pecking sounds, as the bills of the chicks struck the bottom of the basket, could be distinctly heard. The usual mouthing noise made by the mandibles could be heard also when one held his ear near the side of the basket. And when I put my hand into the basket, I could feel the heads of the chicks rising and falling in the pecking reaction.

Before formulating a conclusion let me describe the behavior of no. 7I. This chick, in the pecking reaction tests, made the following record from its second to its eleventh day, and then ceased to peck at the grains when brought to the experiment table:

\section{TABLE 4}

Chick no. 71. Dec. 3-12, 1908. Pecking

\begin{tabular}{|c|c|c|c|c|c|c|c|c|c|c|}
\hline Date. & 3 & 4 & 5 & 6 & 7 & 8 & 9 & 10 & 11 & 12 \\
\hline & 13 & 2 & 3 & 2 & 2 & & & 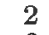 & & \\
\hline & 6 & 25 & 32 & 26 & 22 & 8 & 17 & 6 & 12 & \\
\hline Seized . & & 9 & 5 & 3 & 2 & 3 & 3 & 3 & 1 & \\
\hline Swallowed & 1 & 14 & 10 & 19 & 24 & 39 & 30 & 39 & 37 & \\
\hline
\end{tabular}

On Dec. I 3 it staggered about in a peculiar way, but did not eat. It would walk directly off the table if not prevented. When tested with water in a watch glass, it drank when the water was held to its bill, but after the dish was moved three or four centimeters away, it dipped its bill about where the dish had been. Such indications as these convinced me that the animal was blind. For a day or two it was fed by having grain introduced into its bill. Then it was placed in a large dish partly filled with chick food, and on Dec. 16 it began to scratch and eat. For many days thereafter it was regularly placed in the food dish at the feeding hour and procured its meal without assistance. When I entered the room and the rest of the flock ran toward the wires of the cage, no. $7 \mathrm{I}$ was often seen approaching, running into obstacles in its path, bobbing up and down in a peculiar variation of the pecking movement, and taking time intermittently to scratch in the litter. In this fashion it lived until it was found dead one morning in the drinking vessel. The behavior of the chicks in the dark basket, as well as that of this supposedly blind chick, furnish quite clear evidence in refutation of the assumption that chicks do not peck at or eat food when they cannot see it. 
But happily the reaction in which we are most interested, reaction 4 , can be distinguished from all the others with practically no liability of error. Even supposing a few I's, 2's and 3 's were to become interchanged, the use of reaction 4 as a quantitative index of development would not be invalidated. The main interest is to discover the ratio of the number of perfect reactions to the whole number of legitimate pecking reactions in the daily series, and to plot the variation of this ratio. The necessary data for this can be gathered with precision.

2. Data and their significance. The first animals tested were numbers $I$ to 6 , inclusive, which will be referred to as

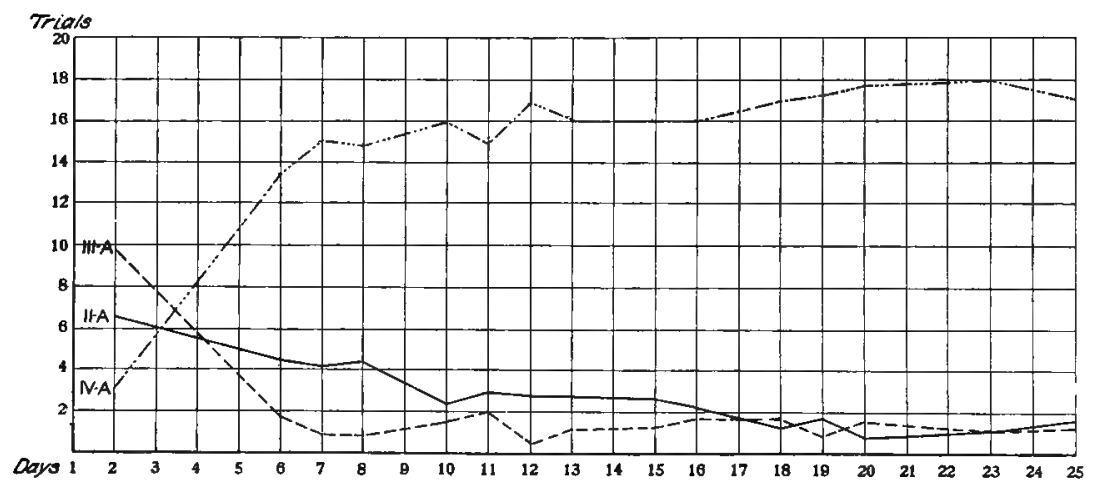

FIGURE 1-The development of the pecking instinct in the chicks of Group A, numbers 1 to 6 , inclusive. Distances along axis of abscissae represent days after hatching; distances along axis of ordinates, the number of a given kind of reaction in a series of twenty pecking reactions. Curve II-A shows the rate of decrease in the daily number of reaction 2; Curve III-A, the same for reaction 3; Curve IV-A shows the improvement in accuracy of reaction 4 for the group.

Group A. They were hatched on Dec. 2, 1907. Their pecking records cover the period from Dec. 3 to 30 , inclusive. Curve IV-A, fig. I, shows the development of reaction 4 for the group. For the calculations only those totals were used in which every chick had a complete series of twenty reactions. Hence a very interesting part of this curve, namely, the detail for the third, fourth, and fifth days, is lacking. The blanks are due to the failure of some of the chicks on each of these days to respond to the food. Improper regulation of the food supply was the cause. Nevertheless, the results were such as to encourage a continuance of the experiment. Barring the vital omissions at 
the most critical stage of development, the curve on the whole not only indicates that the co-ordination of movements that constitute reaction 4 has a certain period of development, but it roughly marks out that course. The rate of improvement is not uniform from day to day, but is very much more rapid during the first few days than later.

Before discussing reactions $\mathrm{I}, 2$, and 3 of this group, let us be clear as to the meaning of these three reactions and their relation to 4 . In the first place, it should be noticed that the records under $\mathrm{I}, 2$, and 3 may be regarded as error records. That is, under I are listed the errors of striking, under 2 the

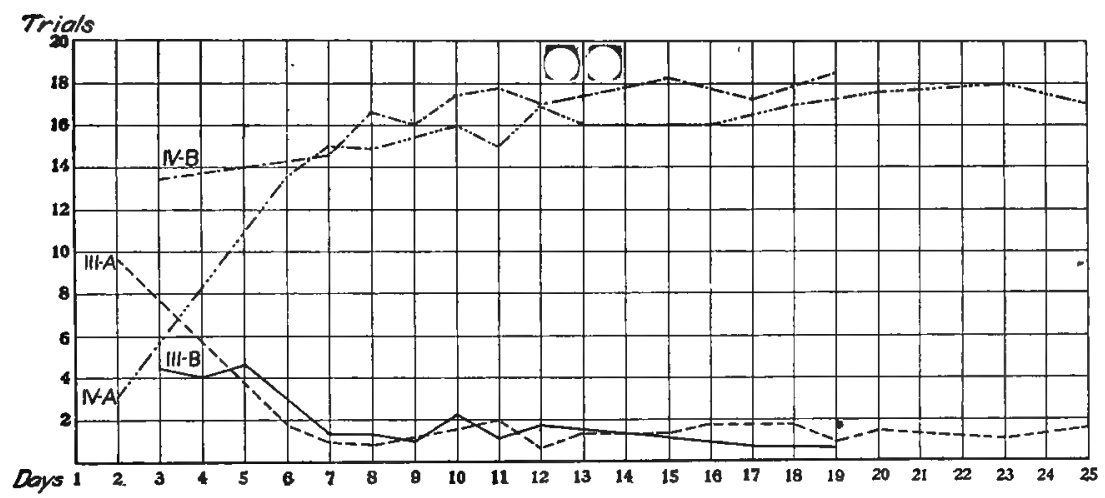

FIGURE 2-A comparison of curves of development of the pecking instinct to show the possible effect of social influence. Distances along axis of abscissae represent days of age; distances along axis of ordinates, the number of a given kind of reaction in a daily series of twenty pecking reactions. Curve IV-A shows the improvement in accuracy of reaction 4, Group A; Curve IV-B, the improvement in accuracy of the same reaction for Group B. Curve III-A shows the course of reaction 3 , Group A; Curve III-B, the course of reaction 3, Group B.

errors of seizing, and under 3 the errors of swallowing, if swallowing here may designate the manipulation of the object after it is seized. Although for convenience the term swallowing has been applied to reaction 4 , it is clear that reaction 4 is really striking-plus-seizing-plus-swallowing in an errorless train.

There are always three regular chances of error, then, at the beginning of any pecking reaction. I shall aim to show the rate of decrease of each kind of error separately, and, correlatively, the improvement in the co-ordination of the three reactions. 
Curve II-A, fig. I, shows the course of reaction 2, and Curve III-A of reaction 3, for the chicks of Group A. The errors of swallowing tend to vanish more rapidly and more completely than those of seizing. The data on reaction I are too meager to justify consideration here.

Curve IV-B, fig. 2, is the curve of development of reaction 4 in Group B, chicks nos. 9 to I3, inclusive. These chicks were hatched just eight days after those of Group A. The two groups were segregated until chicks nos. 9 to 13 were beginning their seventh day, and then were allowed to mingle. The pecking tests were made throughout in the regular way, both groups being treated as nearly alike as possible. Curves IV-A and IV-B, fig. 2 , are matched for corresponding days in the lives of the chicksthat is, for corresponding ages, and not for the dates on which the records were made. Although it was not part of the plan, when the experiment was conceived, to study social influence, the higher curve for Group B indicates that possibly association with the older chicks had the effect of facilitating improvement in the younger ones, and suggests a method of measuring this influence. A glance at Curve IV-A, fig. 2, shows that the efficiency of the chicks of Group A on their fifteenth day, when those of Group B joined them, was numerically $\mathrm{r} 6$ on a scale of 20 , and that the efficiency of Group B at the same time, the seventh day for the latter, was I4.6. But the difference in the curves may be due to unsuspected irregularities in method, or to variability in the chicks. It seemed hazardous, therefore, to rest such an important conclusion on one test, and a repetition of the experiment was planned and executed.

Curves IV-A and IV-B indicate, then, that there is a very rapid improvement in the integration of the components of the complete reaction within the first three days, followed by a slower but fairly steady improvement after that for some time. It is interesting to note just how nearly the development approximates perfection, - I 8.4 on a scale of 20 representing the degree of approximation at the highest point.

Curves III-A and III-B, fig. 2, trace the course of reaction 3 for Groups A and B respectively.

The study of the pecking reaction was resumed almost a year later with chicks nos. 57 to 65 , inclusive, which we shall style Group C. These chicks were hatched Oct. 25, I908. With a 
desire to continue the study of the effect of association of younger with older chicks, eggs were set with the intention of having another brood eight days after Group C, so that the difference between the ages should correspond exactly with the difference in age between the chicks of Groups A and B. But the plan was frustrated to this extent, that the chicks of Group $C$ came out of the eggs one day before the date on which they were scheduled to arrive, and a sudden change of temperature so chilled the second setting that only two healthy chicks arrived to constitute Group D. It was decided to make the most of the opportunity, however, and records were taken of the pecking

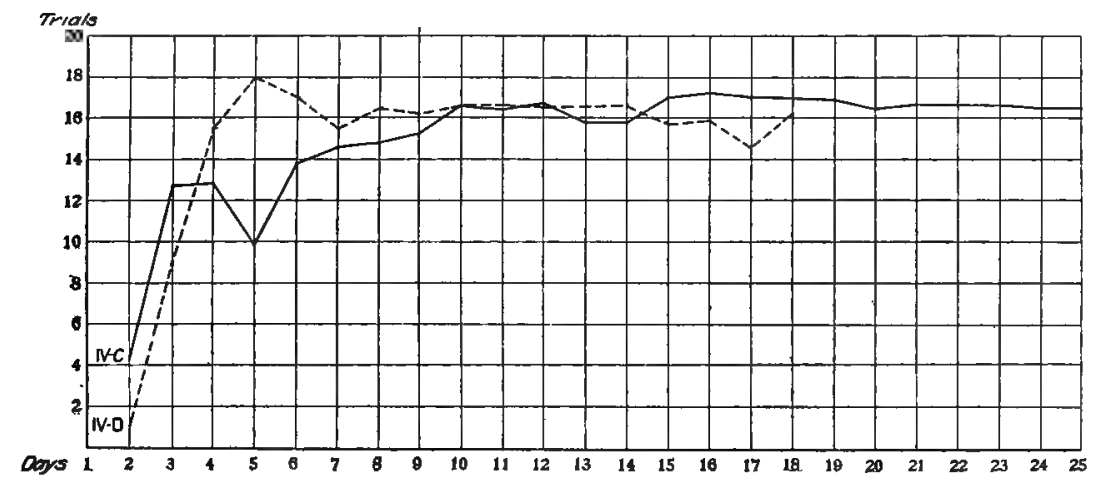

FIGURE 3-A comparison of curves of development of the pecking instinct to show the possible effect of social influence. Distances along axis of abscissae represent days of age; distances along axis of ordinates, the number of "perfect" reactions in a daily series of twenty pecking reactions. Curve IV-C shows the improvement in accuracy of pecking in Group C; Curve IV-D, the improvement in Group D.

of the nine chicks in one group and the two in the other. When the members of Group D were beginning their eighth day, the cages of the two groups were thrown together and the chicks allowed to mingle. For three days, beginning with this day, roo pecking reactions per day were recorded for each chick in both groups; and, continuing for a week after this, 50 reactions a day were taken. It was thought that by this means the influence, if any, of one group upon the other might be more easily and accurately detected. The results are plotted in fig. 3, and the curves matched for corresponding ages as before. The chicks of Group C had a pecking efficiency of I 7.I at the beginning of their seventeenth day when those of Group D joined 
them. On this same date, when the individuals of Group D were eight days old, they had an efficiency of 16.5 . Very little change occurred in the accuracy of pecking of Group D after the commingling, nor indeed could a heightening of their curve after the eighth day be well expected, for they had by that time practically attained the maximum.

Nothing very conclusive having accrued here, it was deemed desirable to arrange a test so that younger chicks could be enclosed with older ones immediately after hatching. Thus, it was thought, the influence might be measured, if the effect of social influence be such as to affect the rate of improvement of the pecking reaction. The most active opponents of inferential, ideational, or voluntary imitation seem agreed that the presence of one animal, under the proper conditions, may have the effect of stimulating another of its kind to greater activity. There is no difficulty whatever in establishing this fact. My observations have proved it to my satisfaction many times. It may be that the change in the mode of functioning of the organism due to the presence of others is in the direction of increasing the intensity and rapidity of the reactions, without increasing their rate of improvement in accuracy. Such a variation would have selective value, inasmuch as the animal would get more food in a given time, even though the pecking were no more accurate. But such a speculation does no more for us than open up wider vistas of experimental research in which, exact quantitative studies can no doubt be made.

Mention has previously been made of the rapidity with which reaction 4 improves. The curves all show very rapid improvement on the second day. To make a special measurement of the rate of this change, the pecking of six chicks in Group $\mathrm{C}$ was tested twice on the second day. The first record was taken from $10-12 \mathrm{a} . \mathrm{m}$. and the second from $2-4 \mathrm{p}$. $\mathrm{m}$. In the interim the chicks were committed to the brooder and allowed the freedom of the litter of the cage for the first time. From forenoon to afternoon, within the limits just stated, the improvement was $82 \%$, assuming, as we have, that the development of reaction 4 is an index of the improvement of the chicks' pecking efficiency.

The sudden drop in Curve IV-C, fig. 3, on the fifth day is due mostly to the unusually poor records. of no. 57 and no. 58 
on that date. Twelve of no. 58's striking reactions were at one particle. It followed this up, hitting it time after time until the grain was knocked off the table.

The final test of social influence in its relation to pecking was made on two comparable groups of six chicks each, which will be referred to as Group E and Group F. The chicks of Group $\mathrm{E}$ were hatched on Dec. 2, I908; those of Group F on Dec. I 2, r 908. The members of Group F, thus ten days younger, were transferred from the incubator to the cages and brooder occupied by Group E. Records were taken as before. Still greater precaution was observed as to food supply. The food was not

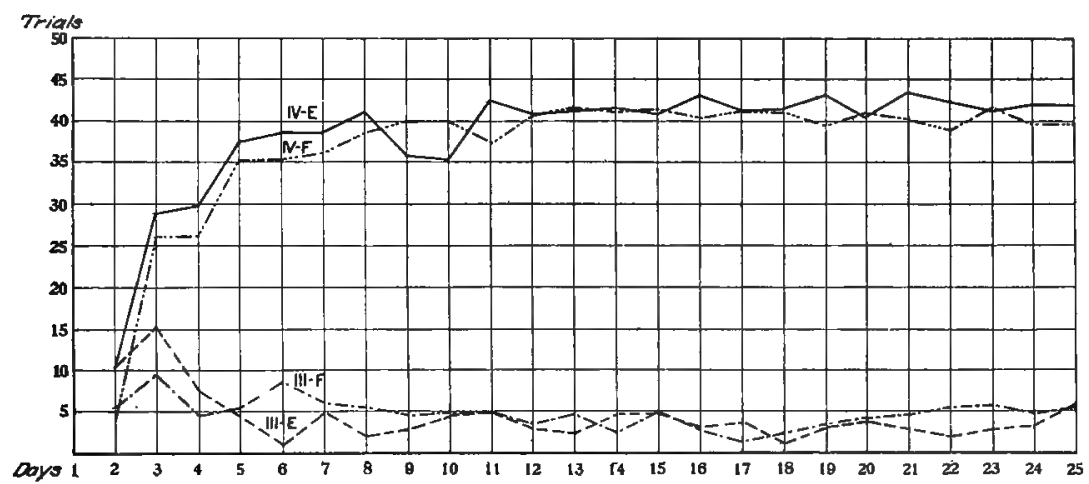

FIGURE 4-A comparison of curves of development of the pecking instinct to show the possible effect of social influence. Distances along axis of abscissae represent days of age; distances along axis of ordinates, the number of a given type of reaction in a daily series of fifty pecking reactions. Curves III-E and III-F show respectively the rate of decrease in the number of reaction 3 for Groups $\mathrm{E}$ and $\mathrm{F}$; Curves IV-E and IV-F show the improvement in accuracy of reaction 4 for the same groups.

unnecessarily stinted, however. The chicks chirped loudly when I came into the room in the morning and crowded toward me when I approached the side of the brooder. In the tests they picked up the grains very energetically.

The records for Group $\mathrm{E}$ are given in full in table 5, the summary for Group $\mathrm{F}$ in table 6. The curves for reactions 3 and 4 of both groups are plotted in fig. 4 ; for reactions $I$ and 2 in fig. 5. The efficiency of the pecking of the older group is represented numerically by 42.2 on a scale of $5^{\circ}$ on their eleventh day, when the chicks of Group F entered the cage. The twelve chicks were confined in the brooder each evening together, were 


\begin{tabular}{|c|c|c|c|c|c|c|c|c|}
\hline & $\mathscr{\&}$ & Ho유 & -No⿱ & N@斿 & $N=\mathbb{B}$ & $-\infty \%$ & +mঞ & 然 \\
\hline & a & $\angle 0 C O$ & $\forall \infty \%$ & $\neg \quad$ 왐 & $10 \infty \frac{\widehat{m}}{2}$ & 요 & $A-\frac{12}{4}$ & त̈ \\
\hline & ন & のッー & 에우 & $\neg N$ F & $00 \%$ & Hभश & $N N F$ & ลี \\
\hline & $\mathscr{N}$ & H $\mathscr{P}$ & 一⿻上丨 & ov $\stackrel{\infty}{\#}$ & $-\infty m$ 앙 & ๒ळ & 요 & ฟิ \\
\hline & నิ & NNO & Nof & $r \quad$ P & $-\infty \infty \infty$ & 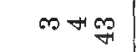 & $-\infty \&$ & $\vec{N}$ \\
\hline & $\bar{N}$ & 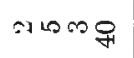 & 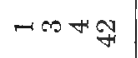 & N & 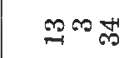 & A & $2000 \%$ & శి \\
\hline$\stackrel{8}{\circ}$ & 오 & NON क्ष & $n m$ 우 & HNZ & $\infty \sim \mathcal{F}$ & வ & $\infty \infty$ & 옥 \\
\hline की & $\stackrel{\theta}{=}$ & $0-$ 암 & $+\infty \quad \infty$ & $\because N G$ & $-\infty \infty$ & DON & 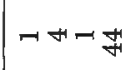 & $\stackrel{\infty}{\sim}$ \\
\hline $\begin{array}{l}\dot{0} \\
\dot{\theta}\end{array}$ & $\stackrel{\infty}{\rightarrow}$ & -cHs & $-\infty \infty \infty$ & $\infty \frac{\gamma}{y}$ & ーナおも & $\infty \forall \Re$ & $N N F$ & $\approx$ \\
\hline ت્9 & $\approx$ & מN & H以 F & $7<0$ F & ๓⿴⿱冂一⿰丨丨八 & NH & m- & 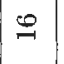 \\
\hline 5 & $\stackrel{\bullet}{-}$ & 1010 의 & $-\infty \in O$ & NलS & $\infty 00$ & $\infty \times \underset{f}{*}$ & $\mathscr{O N} \underset{Y}{ }$ & 10 \\
\hline 陆 & $\stackrel{10}{-1}$ & サッே & $7+\infty \cos$ & 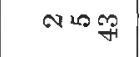 & $\neg-\infty \mathbb{W}$ & ம & $\infty \stackrel{\infty}{\stackrel{+}{*}}$ & $\exists$ \\
\hline$R^{2}$ & $\#$ & $\forall N-\infty$ & - $10 \%$ 유 & 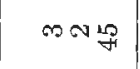 & $n \infty$ O & $\sim \stackrel{\infty}{\#}$ & $-\infty g$ & 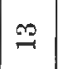 \\
\hline z & $\stackrel{\infty}{\rightarrow}$ & $\infty N \leadsto \infty$ & ON & 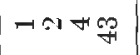 & $\cos$ 연 & ヘーঙ্ & 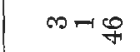 & $\cong$ \\
\hline$\frac{0}{2}$ & $\mathcal{Y}$ & $\neg \infty \times \frac{y}{y}$ & N०\% & $\infty$ & $\infty \infty$ & - & $N \odot$ F & $=$ \\
\hline $\sin$ & $\Rightarrow$ & $\infty \pi$ & $\infty \mathrm{Non}$ & $-\infty$ & - 120109 & HNg & $\vec{\infty} \stackrel{\sigma}{\sim}$ & 웅 \\
\hline$\hat{R}$ & 옥 & $\infty+\infty$ & $\forall \rightsquigarrow \dddot{F}$ & NH出 & $\infty$ N유 & $\operatorname{sn}$ & ब्रक & 0 \\
\hline 8 & $\sigma$ & NलI & $+\Leftrightarrow \underset{H}{H}$ & \& & $\cong-\mathscr{్}$ & $A-72$ & $\stackrel{\sim}{N}$ & $\infty$ \\
\hline $\begin{array}{l}0 \\
0 \\
2\end{array}$ & $\infty$ & 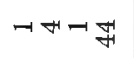 & nog & $\operatorname{Tr}$ & Nᄋ్తా & ๓⿴⿱冂一三丨 & 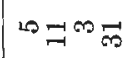 & $N$ \\
\hline 兽 & $N$ & N+g & $10 \mathrm{cos}$ & H & $\stackrel{H}{\sharp}$ & $-7=-1 \times$ & - & 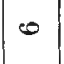 \\
\hline 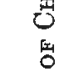 & $\varnothing$ & NorF & $N \forall$ & $-1-1$ & $\stackrel{\infty}{-} \underset{\sim}{\sim}$ & $\infty$ 음 & 앳⿵冂卄 & 10 \\
\hline 总 & 10 & $-\infty$ 1 & Nমㅓㅛ & $\cos \infty$ & $N=$ & $\cong \Xi ゙$ & NFg & 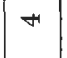 \\
\hline 窇 & $H$ & $10+7$ & 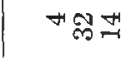 & 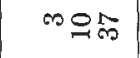 & -NN & 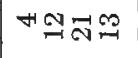 & $a r e$ & $\infty$ \\
\hline & $\infty$ & $\infty N+\pi$ & $\infty \infty_{-\infty}^{\infty}$ & H & 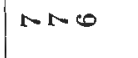 & D סמ & $\forall m$ & N \\
\hline & 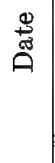 & 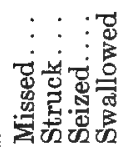 & 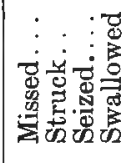 & 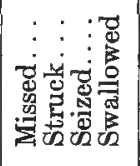 & 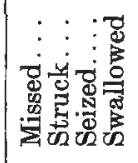 & 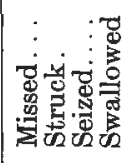 & 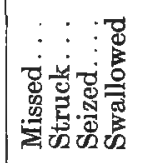 & 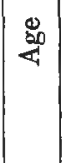 \\
\hline & se & 8 & 요 & N & $\Re$ & 12 & $\stackrel{R}{1}$ & \\
\hline & ت्: & $\dot{8}$ & 安 & 完 & $1 \dot{0}$ & i & $\dot{0}$ & \\
\hline
\end{tabular}




\begin{tabular}{|c|c|}
\hline \#品昂 & $\ddot{\sim} \dot{0}$ \\
\hline 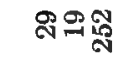 & ن \\
\hline 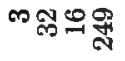 & مِ \\
\hline 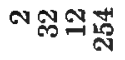 & ๓ฺ \\
\hline 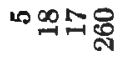 & $\infty$ \\
\hline 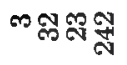 & مَ \\
\hline 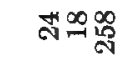 & सं किष \\
\hline 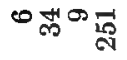 & 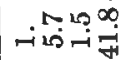 \\
\hline m呤导 & لَ \\
\hline 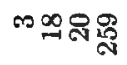 & 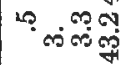 \\
\hline 一弎坛 & N \\
\hline N只穴 & 䒠 \\
\hline 以 & 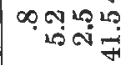 \\
\hline +M̃ & نـ \\
\hline 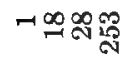 & ฺִ \\
\hline - & 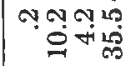 \\
\hline 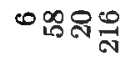 & تص \\
\hline 同舀思 & \\
\hline$\infty$ సే心 & 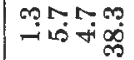 \\
\hline N & مَ \\
\hline 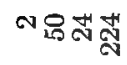 & ڤִ \\
\hline 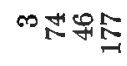 & 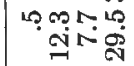 \\
\hline 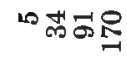 & 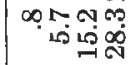 \\
\hline 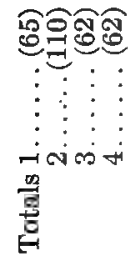 & 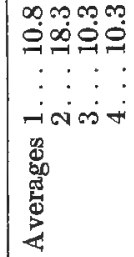 \\
\hline
\end{tabular}

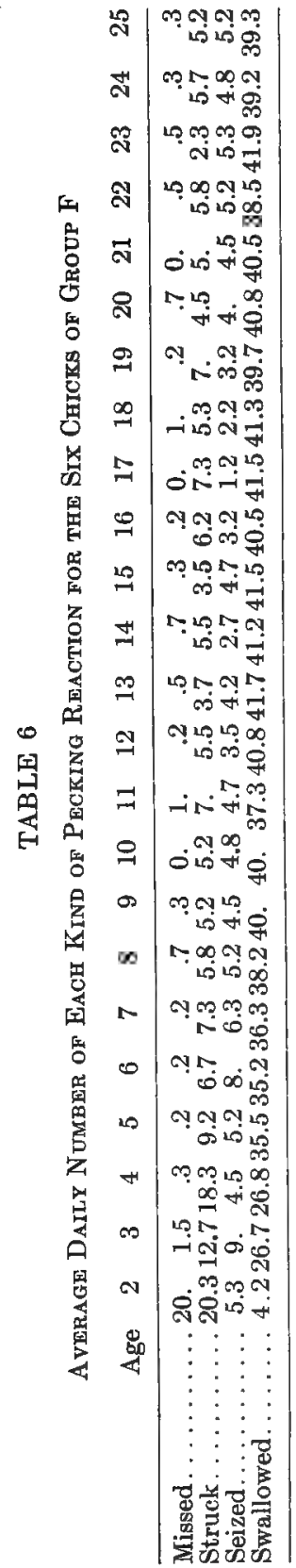


given their tests at the same period in the morning, and of course were fed together in the same litter, both groups being compelled to scratch for their food. Yet the similarity of the curves for the first eight days, when modification progresses most rapidly, is conspicuous, the curve for the younger brood running slightly lower. These young chicks, in spite of the examples of more accurate pecking furnished them by the behavior of the chicks of Group E, began less accurately than their elders, remained behind them by about the same margin during the critical period of development, and hardly equalled them while the experiment continued.

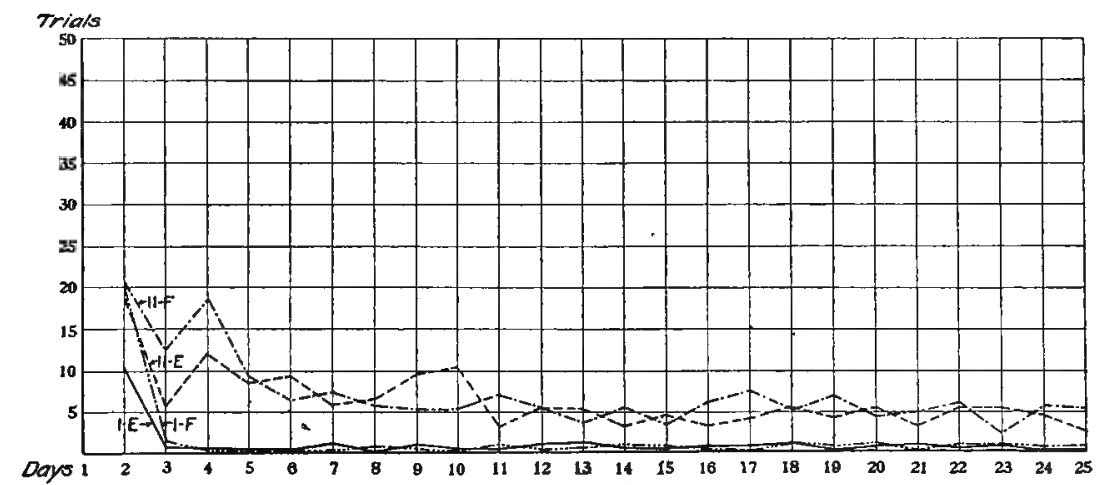

Figure 5-A comparison of the curves of development of the pecking instinct to show the possible effect of social influence. Distances along axis of abscissae represent days of age; distances along axis of ordinates, the number of a given type of reaction in a daily series of fifty reactions. Curves I-E and I-F show the rate of decrease in the number of reaction 1 for Groups $\mathrm{E}$ and F, respectively; Curves II-E and II-F, the rate of decrease in the number of reaction 2 for the same groups.

A feature worthy of notice is the fact that development of reaction 4 in both groups seemed to halt on the third day, with very rapid improvement preceding and following. A search for the cause of this retardation of development in the chain of actions is interesting. A detailed examination of the relations of Curves I, II, III, and IV for both groups brings out the following points:

Ist day, the course of development is unknown;

and day, striking and seizing improve rapidly, while swallowing (in the restricted sense) declines considerably in effectiveness; 
3rd day, swallowing improves rapidly, striking improves slightly, and seizing suffers a reversal of form.

In other words, from the beginning of the second day the accuracy of striking improves uninterruptedly, the accuracy of swallowing declines temporarily on the second day, and that of seizing temporarily on the third day. It is evident that the rate of improvement of reaction 4 depends upon the rate of decrease of the total number of errors in these reaction types. In neither Group $\mathrm{E}$ nor Group $\mathrm{F}$ was improvement in all three types of reaction uninterrupted. The retardation of reaction 4 on the third day is due specifically to a decline in the accuracy of seizing on that day.

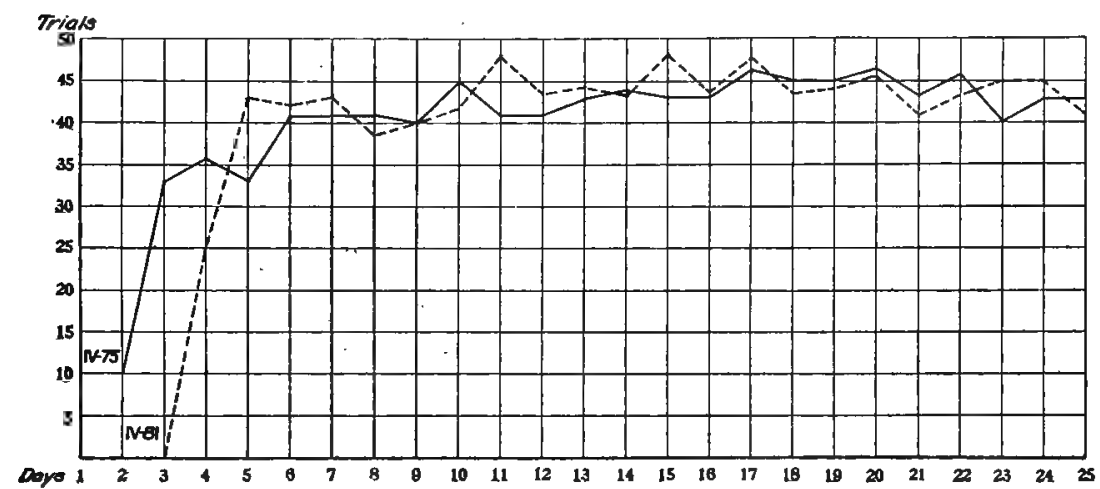

Figupe 6-Curves showing the rate of improvement in pecking accuracy of two chicks kept in isolation. Distances along axis of abscissae represent days; distances along axis of ordinates, the number of perfect reactions in the daily series of pecking reactions.

In regard to the tests for social influence, the objection can be made that social influence, if it affect the accuracy of reaction at all, may work as well between members of the same group as between members of different groups. The objection has force. As a control test, chicks nos. 75,80 , and $8 \mathrm{x}$ were kept'in individual apartments and tested. The results are exhibited in fig. 6. The record of no. 80 is omitted because valueless. This chick soon became physically unfit. No. 75 , although it did not thrive under the conditions, made a good pecking record. No. 8I not only pecked with unusual accuracy but was lively and vigorous after the first few days. The curves do not furnish convincing evidence in support of the view that social 
influence accelerates improvement in the accuracy of the reaction studied.

The curve for no. 8r clearly suggests retardation of development--a very interesting fact, if due to the isolation. At first this animal seemed quite indifferent to food. Besides, its toes were bent under in such a way that it looked crippled. In a few days, however, neither of these failings was observable. They may indicate that the chick was slow in the process of unfoldment in more respects than in pecking. It would hardly be fair, for example, to attribute the slow expansion of the toes to the isolation. No. 8I turned out to be a fine animal. The rapidity with which the components of reaction 4 became integrated, once the process set in, is instructive. The maximum of accuracy attained, as well as the high average maintained, mark the nicety of adjustment in no. 8I's pecking mechanism.

If chicks that are isolated peck with a normal degree of accuracy within the natural time limit, and chicks stimulated by the presence of others much more efficient than themselves fail to show a supernormal rate of improvement, one has good grounds for believing that the social influence, whatever else its effect, does not appreciably modify the natural course of development of the pecking concatenation.

In fig. 7 are presented the curves of development of the pecking reaction, based on the averages obtained from the records of the twenty-one chicks in Groups $\mathrm{C}, \mathrm{E}$, and $\mathrm{F}$ during their first twenty-four days. The data for these curves are given in table 7. Curves I, II, and III, standing for reaction I, 2, and 3 , respectively, represent, as explained before, the distribution of errors. Curve IV represents reaction 4. The sum of the heights of the error curves above the base line on any given day will equal the distance of Curve IV below its limit for the same day.

"In nearly all cases, as one might expect," says Morgan," "the simple process of striking is more accurate than the more complicated process of striking and seizing; and this, again, than the yet more elaborate process of striking, seizing, and swallowing." From the data in table 7 it is an easy matter not only to substantiate this assertion, but to show the quanti-

\footnotetext{
${ }^{1}$ Morgan, C. L.: Habit and instinct. London and New York, 1896, p. 37.
} 

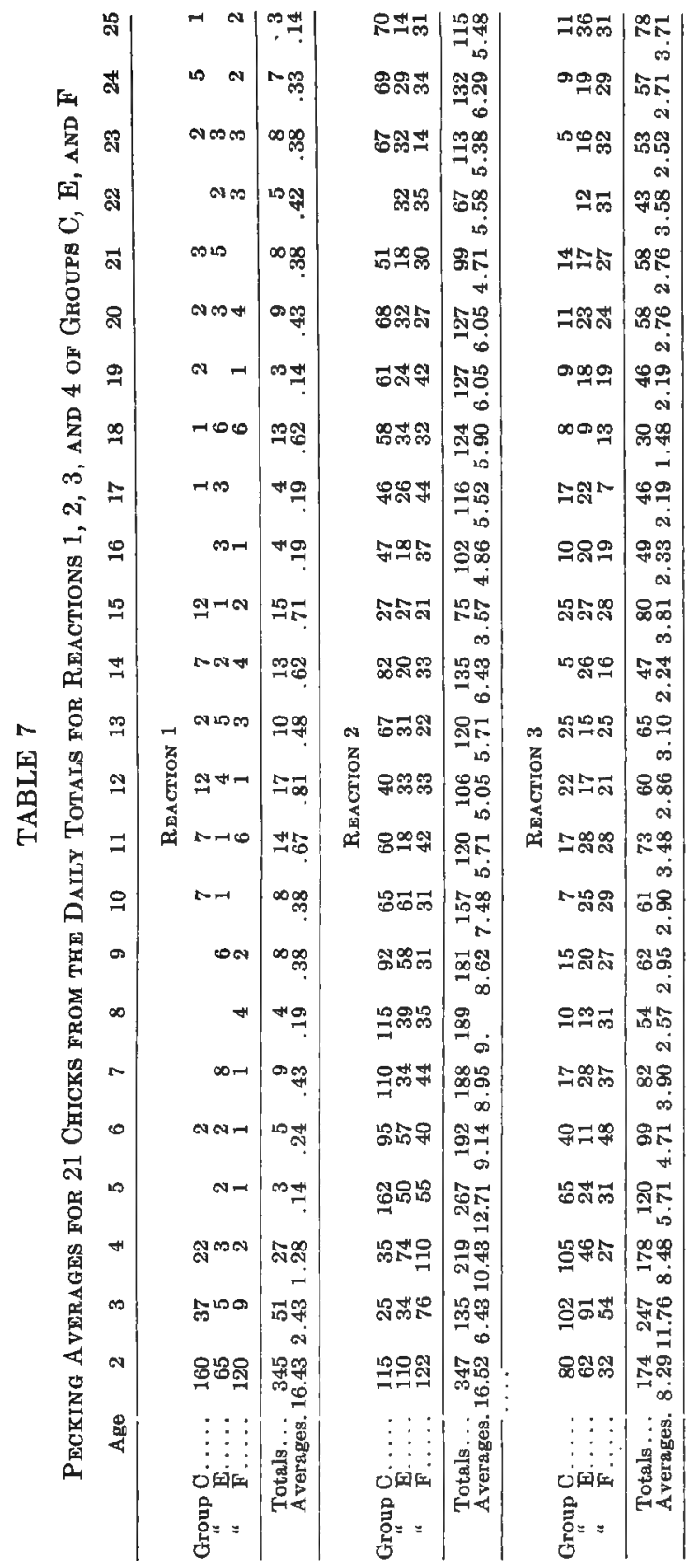

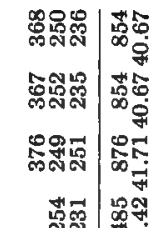

药

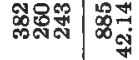

율츄

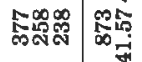

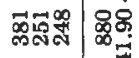

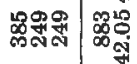

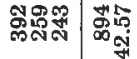

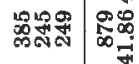

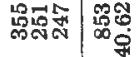

+

然

萭

ตัสส

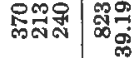

ิㅗㅊㅠㅠ

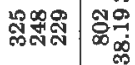

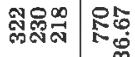

궁죽ㄱ

สิสีสี

녹효

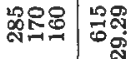

หูำํำ

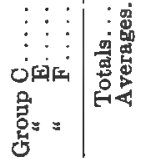


tative relations involved. E.g., on the second day, out of fifty trials the object was struck, on an average, 33.48 times on the first attempt. Of this number only $\mathrm{r} 6.96$ included seizing; and of the latter only 8.67 were reactions in which the object was struck, seized, and swallowed. As the chicks advanced in age, each of these sets of figures approximated the same limit, but naturally they preserved the original order as to size, for with each additional reaction in the series there was an added source of error.

Reverting to the earlier consideration that Curves I, II, and III may be regarded as error curves, an examination of these

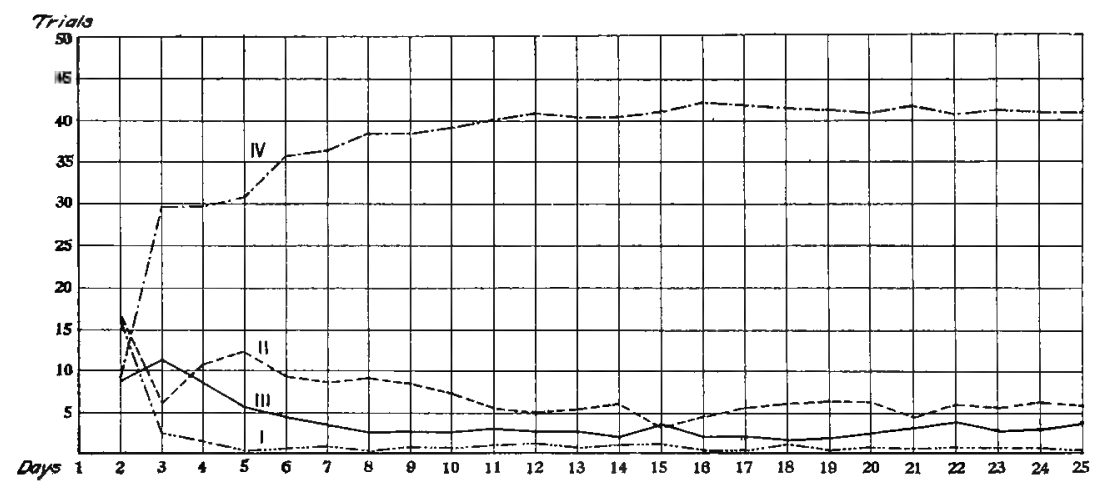

Figure 7-Curves of development of the pecking instinct, based on the averages obtained from the records of twenty-one chicks. Data in table 7. Distances along axis of abscissae represent days of age; distances along axis of ordinates, the number of occurrences of a given type of reaction in a daily series of fifty pecking reactions. Curves I, II, and III trace the course of reactions 1,2 , and 3 , respectively. Curve IV shows the average improvement in accuracy of reaction 4.

curves shows that the improvement of pecking accuracy is retarded more by failures in seizing than by failures in swallowing; and more by failures in swallowing than by failures in striking. After the third day, the difficulty of seizure remains greater than that of the other two reactions combined. The striking reaction improves in accuracy rapidly and without relapse, closely approximating perfection by the fifth day-a degree of accuracy that might easily inspire belief in the perfection of instinct, for, indeed, this is the reaction which has no doubt been central in the earlier discussions when accuracy has come into question. 
Of course these averages hide the unusual performances of individuals. Chick no. 72 , for instance, missed only once out of fifty reactions on its second day, on the fifth day it struck, seized, and swallowed forty-eight grains in fifty trials, and on the eighth day devoured every one of the fifty without a slip. The complete record of this animal is given in table 5. A comparison of its record with that of no. 70 brings out nicely the point of individual differences in accuracy. No. 72 developed more rapidly than no. 70, and also attained a much higher average degree of efficiency. The records of both chicks are consistent throughout. They bespeak a more finely adjusted mechanism in the one case than in the other. If we were seeking points of difference instead of identity in this investigation, we should dwell more at length an variations in other respects. Chicks, as one might expect, differ definitely and consistently from day to day in the accuracy of their reactions, in the rapidity of their movements, in their food preferences, and the like, much as men do.

To return to fig. 7. The curve representing the course of development of the complete co-ordination, Curve IV, reaches its highest elevation during the twenty-four days at 42.5 on a scale of 50. Each of these curves is intended to represent an aspect of the pecking of the chicks under the conditions described. Whether or not the reactions in the litter were more accurate than those on the harder cardboard, I cannot say, not having made any measurements. To be sure, the long periods of practice were spent in pecking under conditions quite different from those that obtained in the tests.

It has been the aim of this investigation to discover the meaning that should attach to such expressions as the accuracy, the perfection, or the congenital definiteness of the instinctive action that has been generally considered one of the most perfect, namely, the pecking reaction of chicks. Morgan and Thorndike are correct in asserting that this reaction is imperfect at birthnot "very nearly" perfect as Morgan thinks, but very imperfect. But Thorndike ${ }^{1}$ doubts the fact of improvement. "As a matter of fact," he writes, "the pecking reaction may be as perfect at birth as it is after 1o or I 2 days' experience." I shall have to

${ }^{1}$ Thorndike, E. L.: The instinctive reaction of young chicks. Psych. Rev., 1899, vol. VI, p. 285. 
side with Morgan here and insist that the pecking reaction improves in accuracy after birth. Whether, now, this is a case of perfecting through habit, involves a still further problem, that of the relation of habit to the instinct. One sometimes speaks of the modifiability of an instinctive action like that of pecking, but wherever this term has been employed in this paper in connection with instinct no more has been implied than the objective fact of improvement in accuracy, an increasingly successful adjustment of parts in a more comprehensive function. The problem still remains, Is this development dependent upon practice, or is it the natural functional correlate of structural maturation independent of practice? Swallows are reported to be able to fly without previous practice. If the pecking of chicks could be successfully inhibited for a week's time without doing violence to the normal physical condition of the animals, would the accuracy of the reactions at the end of that time average 36.67 on a scale of 50 , the average for our lot of twentyone? There is evidence in support of the belief that such chicks would very quickly be pecking with average efficiency, without anything like the amount of practice chicks would have had by this time when growing naturally. In other words, improvement does not depend entirely upon practice. How much of the improvement does depend upon practice? All of it, one is led to believe from Morgan's pages. "Steadying of the inherited organic apparatus " through preparatory efforts means improvement through habit. Besides, overestimating, as he did, the degree of perfection of the instinct at birth, he has left less room for maturation and the effects of practice than there really is. So far as the facts are concerned, the most one can say is that the development of the pecking instinct proceeds somewhat without practice and is hastened by it. Maturation and use run along in time together. No means has yet been devised of measuring the amount either factor apart from the other contributes to the development of the pecking reaction.

The importance attached to individual acquisition as a factor in development seems on the whole to become increasingly restricted. The theory of the non-transmission of acquired characters enormously narrows its scope in phylogenetic development. Perhaps we shall discover its lesser importance in ontogenetic development. The drinking reaction, for example, is 
more self-dependent than we have hitherto supposed. The pecking reaction suffers the supplementation of habit, one may well believe, when it does not demand it. It remains to be shown to what extent these instincts are typical of instincts in general. But even if it should be established that acquisition contributes relatively little, environment would still remain a powerful factor in development. The animal begins life with an hereditary endowment in interaction with the environment. One is necessary to the other. In the economy of an organism there is no reaction without stimulation. And not only is the environment a system of energies without which natural tendencies of the organism cannot be realized, but it is a selective system. What tendencies shall be realized will be determined by what stimuli are provided. Heredity and environment are not opposites, but complements.

\section{PART II. ACQUIRED REACTIONS}

\section{Introduction and Statement of the Problem}

From the study of the modifiability of reactions that are made prior to experience and are supposed to be based on inherited neuro-muscular co-ordinations, our interest now shifts to a study of the development of reactions that depend upon no such hereditary dispositions in the nervous system, but are individually acquired. It was one of the aims of this investigation to discover not only whether reactions to certain optical stimuli or stimulus-complexes are modifiable, but also to determine the rate of modification; in other words, to describe the progress of habit-formation in quantitative terms.

Modifiability was tested by the so-called discrimination method. Never more than two possibilities of selection were offered to the animal at one time. The conditions were so arranged that the chick's natural tendency to react to confinement and solitude by efforts to escape furnished the necessary random activity. When, after a certain number of trials, the animal reacted selectively to one of two constant form or size or color stimuli, for neither of which it displayed a preference before training began, the process of habit-formation was adjudged completed. -By selective reaction is here meant the ability to react without error to a constant stimulus for an arbitrarily fixed number of times. 
The adoption of the discrimination method followed directly from the results of Yerkes' work with the dancing mouse. I have appropriated his method, my contribution being merely an adaptation of the method to the study of chicks. So much for the problem in a general way.

\section{IT. Apparatus}

The apparatus used was a fan-shaped reaction box built of wood and painted brown. Fig. 8 shows the ground plan, fig. 9 the perspective. The method of construction was as follows. On a base 88 by $95.5 \mathrm{~cm}$. a convenient point near one edge was selected from which as a center, with radii of $38.64 \mathrm{~cm}$. and $69 \mathrm{~cm}$., respectively, arcs of circles were described. This center later became the point from which the chick started at each trial, and around which the entrance box was built. The nearer arc marked the location of the cards first approached by the chick; the more distant one outlined a boundary of the apparatus. The box was made with four compartments, but only the two inner ones were used in these experiments. Removable partitions (J, fig. 8) made possible this use of only a limited portion of the box. The height of the apparatus was $20 \mathrm{~cm}$., inside measurement. The covering consisted of two lifting doors, one over the entrance box A and the other over the rest of the apparatus. These were made of light wooden frames and wire netting, and were so arranged that they lifted on their hinges directly away from the experimenter as he stood at the entrance box. The mesh of the netting was $3 \mathrm{~cm}$. - as large as conditions would permit, so as to interfere with the light as little as possible. The exits $\mathrm{H}$ were closed with removable galvanized sheet iron sliding doors that fitted from above in vertical grooves. The end of the box about $G$ was closed with wire netting of $.86 \mathrm{~cm}$. mesh. Grooves at this end also provided for wooden screens inside the wire of sufficient height to conceal the flock of chicks in a cage just beyond and below the experiment box from the chick reacting. Two sets of cards were used, one at $D$ and another at $F$. The card-carriers at $F$ projected $2.55 \mathrm{~cm}$. from the walls, making it impossible for the exit $\mathrm{H}$ to be seen from the chamber $\mathrm{C}$.

Following the ground plan in fig. 8 and the path of a chick through the apparatus from the entrance box $A$, the remaining 
dimensions and details may be clearly supplied. From the entrance box A, I6 by $20 \mathrm{~cm}$., the chick was released by a wire screen door of $.86 \mathrm{~cm}$. mesh that was raised and lowered in its grooves at $\mathrm{B}$ by the experimenter. This retaining screen was shaped so that its lower edge coincided with an arc of a circle constructed from the center above referred to. The width of the opening at $\mathrm{B}$ was ro $\mathrm{cm}$. The first cardholders were at $\mathrm{D}$, the passage ways through which were $9.33 \mathrm{~cm}$. wide. Through

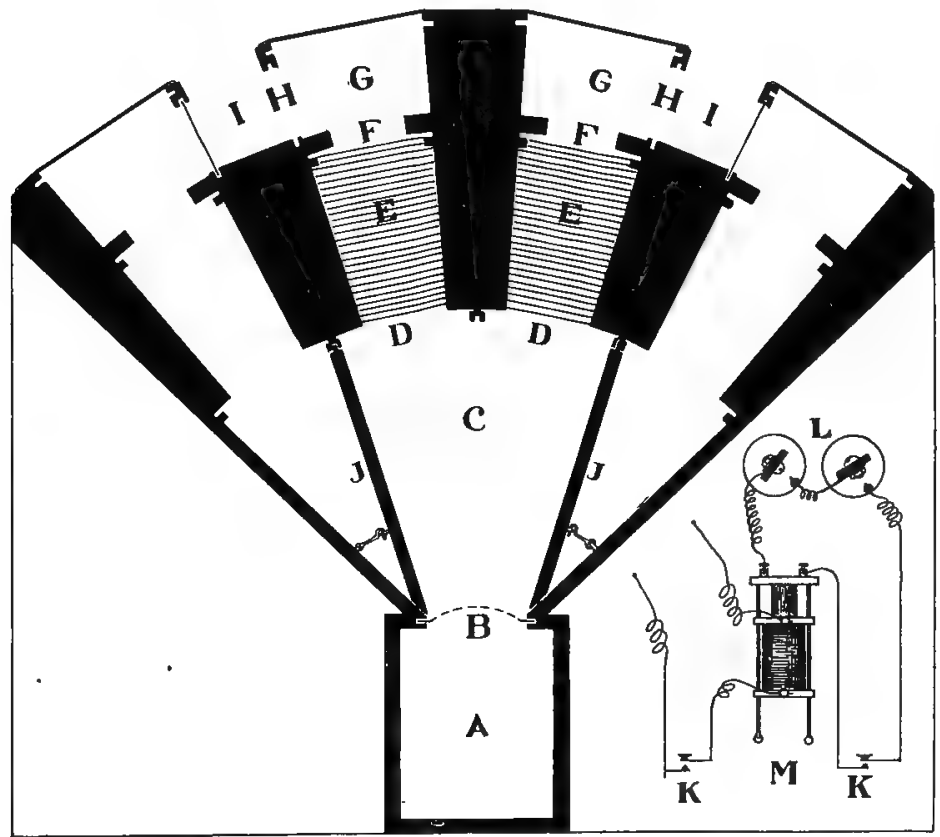

FIGURE 8-Ground plan of apparatus for testing habit-formation. A, entrance box; $\mathrm{B}$, wire-screen door; $\mathrm{D}$ and $\mathrm{F}$, location of card-holders; $\mathrm{E}, \mathrm{E}$, electric chambers; $G, H$, I, way out of box; $K$, electric key; L, electric battery; M, inductorium.

$\mathrm{D}$ the chick passed into the electric chamber $\mathrm{E}$, the floor of which was crossed with copper wires I $\mathrm{cm}$. apart, so arranged that they formed an interrupted circuit which could be closed by the chick when the current was on by its stepping simultaneously on two adjoining wires. Out of $\mathrm{E}$ the chick passed through the opening F, $8.5 \mathrm{~cm}$. wide, the position of the second card. From $G$ there was an. outlook through the wire netting 
upon the flock of chicks in the cage below and also an outlet through $\mathrm{H}$ which was $8.5 \mathrm{~cm}$. wide. The door of galvanized sheet iron could be inserted here to close the exit when desired. Once in I, the chick joined the rest of the flock by passing down over the door of the cage that was turned up toward this end of the apparatus at an angle of about 45 degrees. The dimensions and details were the same by either of the routes a chick might take from $\mathrm{A}$ to the cage. $\mathrm{K}$ indicates a key by which the circuit was made and broken. The current was supplied by

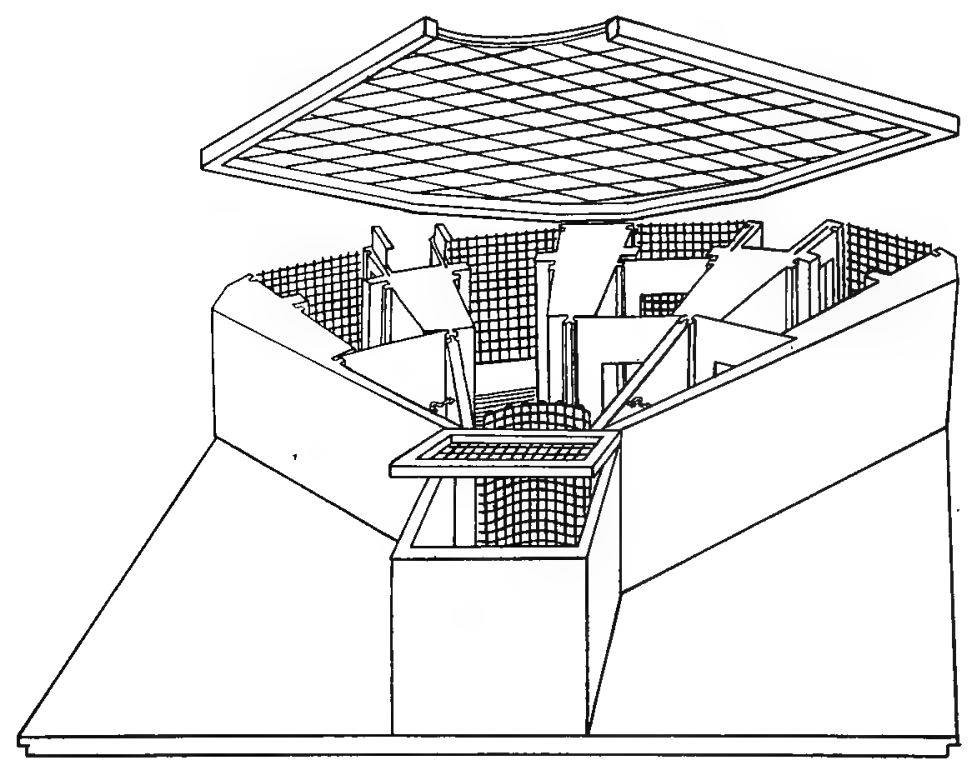

Figure 9-Perspective of apparatus for testing habit-formation, ground plan of which is presented in fig. 8.

a no. 6 Columbia dry cell which was connected with the primary coil of a Porter inductorium, the secondary coil of which was in circuit with the ends of the two wires that were wrapped parallel to each other about floors that were fitted into the electric chambers E. During the tests the apparatus was so placed that the entrance box was nearest the source of light and the sides of the apparatus were symmetrically situated with reference to the two windows that admitted the light. The cards used at D were $14.25 \times 19.5 \mathrm{~cm}$. and those at F $\mathrm{I} 3.5 \mathrm{X}$ 
I8.75 cm. In all color work the openings in the two sets of cards were the same in size, $8.5 \mathrm{~cm}$. wide and II.5 cm. high. These rectangular openings were cut in the lower part of the card in such a way as to leave equal margins of color on either side of the entry way.

\section{IIII. Method}

For a test of the reactions to color, form or size the appropriate cards were placed in the card-holders. For example, if reactions to black and blue were to be tested, one might start with a black card in each of the holders at D and F, right, and two blue cards in the corresponding holders on the left. The cage in which the animals lived was carried to a position with reference to the table upon which the apparatus sat such that one of the wire netting doors of the cage would turn back toward the exit-end of the apparatus as above described, and form an easy passage-way from the reaction box to the cage.

The chick to be experimented upon, marked and numbered, was carried from the cage to the entrance box. When placed in $\mathrm{A}$, it soon acquired the habit of crowding up near the wire netting door, standing at a point equidistant from the two cards exposed at D. It also soon ceased pecking at the wire covering or flying against it, if it happened at first to try this way of escape. The work had not gone very far before the plan was rigidly adhered to of not releasing a chick from $\mathrm{A}$ until it made an effort to escape by urging toward the screen. Sometimes this meant a delay of several minutes, but it seemed useless to spend time with a chick that did not react negatively to the confinement or isolation. Further, care had at all times to be exercised that a chick be not kept struggling in A. Results were vitiated if this occurred. I feel sure that discriminating ability deteriorated as the response to the isolation of the reaction box approached too near in one direction the passivity of indifference, or in the other the activity of excitement. I have watched the record of indifferent chicks recover from a lapse when the response to isolation was accentuated by sufficiently prolonged solitary confinement between trials; on the other hand it is easy to point to numerous instances where a record has been marred by undue excitement. I concluded from experience, that much the wisest plan was to watch for an incipient struggle to escape, and to lift the door just when the 
impulse had begun to gather force. There is a nice middle ground here that gave the best results. There were great individual differences in the animals. Some stood about for a while before the impulse to escape seized them, others rushed immediately toward the screen door. The experimenter stood back of the entrance box between the two windows of the room, and, after releasing the chick from $\mathrm{A}$, remained as quiet and motionless as possible until the animal found its way out of the apparatus. I had practically no success with the device Yerkes used with the dancing mouse, the card by which he gradually forced the animals toward the electric box. Chicks that won't work without force won't work with it, is a rule that can be generally relied upon. After the animal had escaped to the cage, the experimenter secured it again, brought it back to the entrance box, and the procedure as described above was repeated.

At the beginning of a set of experiments the animal was familiarized with the apparatus, as well as tested for its preference of the stimuli presented in the card-holders, by a number of " preference trials," usually one series, which consisted of ten trials. Sometimes a second series was given. These series are denoted in the tables by $\mathrm{A}$ and $\mathrm{B}$, respectively. During the preference trials the exits at $H$ were both left open, the two sets of cards were alternated right and left after each trial, and no electric shock was used. To show the readiness with which the chicks reacted in the preference trials as well as to suggest the availability of the animals for certain kinds of labyrinth training, I present below as typical the average time in seconds for each preference trial, from one to ten, of chicks nos. 32,33 , 38 , and 39 , Group I, at the beginning of their black-blue work: $69.2,24.1$, I8. I, 3.8, 3.4, 2.6, 2.I, I.9, 2.2, I.9. Chicks thus tested for an experiment were continued in that experiment, unless they were found to have a preference for the stimulus to which it was desired they should form the habit of positive reaction. A chick that revealed a preference for this stimulus prior to training was rejected.

After the preference trials had proved a chick satisfactory, it was subjected to the regular conditions of training. The "wrong" side of the apparatus was closed at the exit and the animal was given a shock if it entered the electric chamber on that side. This was done by quietly pressing the key previously referred 
to. The shock was not severe enough to cause the chick to jump from the wires or chirp on receiving it. It was found that a shock from a current value of about 1.69 amperes with the secondary coil set at 5.5 according to the scale on the inductorium, proved most generally satisfactory. Here again there were individual differences in sensitiveness among the chicks, but in the main the above shock brought results without being injurious, and had no more effect on the chicks than to start them from their position, occasionally causing one to lift a foot from the wires. The floor of the entrance box was kept wet so that the feet of the chicks would be in a condition to receive the shock with regularity.

At first a chick usually ran right over the wires and on to the front netting, from which there was a full view of the other chicks in the cage. With the chick in this position on the "wrong" side, it was found best to wait until it happened upon the right road out by its own random activity. Some times the wait on the first training trial was ten minutes, but such a delay was exceptional. The young chicks, as a rule, were active and soon acquired the habit of withdrawing from the "wrong" side promptly. The draft on the experimenter's time after the training was well under way, averaged about I. 5 minutes per reaction, including everything. No trouble was experienced with hesitancy on the part of the chicks to return over the electric wires. Of course they were never shocked on the return passage.

In a series of trials the positive card was shifted right and left in this order: $r, 1, r, 1, r, r, 1,1, r, 1$. Thus each card in each series was displayed the same number of times on the rightand left-hand sides. When the chick had made a series of errorless reactions with the above changes of cards, it was tested for another perfect series with a different order and an equal number of rights and lefts. If no errors were made this time, a third series with still a different sequence of changes was given, when, if the chick reacted without error, the process of habitformation was pronounced completed.

In none of the work with this apparatus was it necessary to starve the chick or even to keep it excessively hungry. It is my opinion that hunger did not play a more important part than the reaction to confinement and solitude. True, it is easy 
to show that, as a rule, a chick with a full crop is not so good material for an experiment like this as one not so well fed, but these animals while being experimented upon did not always rush about for food as soon as they had reached the cage.

\section{Experiments}

In these experiments on modification thirty-eight chicks were used. The work was done from Dec. I5, I907 to June 30, I908. To test the interrelation of habits of response to optical stimuli, the necessity arose of discovering at least two methods of measuring modifiability in this sense mode. It was desirable in the case of any one method that the stimuli bear such a relation to each other that the average chick could complete the training within a reasonable time, and that the time should be as nearly as possible constant for different chicks. The feasibility of the electric shock and satisfactory conditions and devices for training the chicks were determined upon after several hundred preliminary trials in a crude reaction box. The apparatus shown in figures 8 and 9 was constructed on the basis of experience in these preliminary tests, combined with the suggestions afforded by the apparatus of Yerkes ${ }^{1}$ and that of Hamilton ${ }^{2}$ used in similar experiments. Means for retaining the animal in the entrance box until it made movements to escape, permitting it meanwhile to be within range of the stimuli; the location of these stimuli at a suitable distance from the point of release; the open screen overlooking the cage-these features were planned with characteristic activities of the chicks in mind. Releasing the chick too soon or too late at any given trial produced unsatisfactory results. If the cards were too near, the pulse of activity often carried the animal into the electric box before the card stimuli had wrought the proper effect. And when the animal had made an error and had arrived at the closed exit, the chicks in the cage below acted as an additional stimulus to escape. The animals readily found the exit through $\mathrm{H}$ when the door was open.

\section{A. Color}

In all the color work the reflection method with the Bradley standard colored papers was employed. Hue, tint, and shade

\footnotetext{
1 Yerkes, R. M.: The dancing mouse. New York, 1907, p. 92.

${ }^{2}$ Hamilton, G. Y.: A study of trial and error reactions in mammals. The Journal of Animal Behavior, 1911, vol. I, p. 33.
} 
are described only by reference to the catalogue name or number of the papers. Measurement of tint (or brightness) was attempted in only a few special cases. The purpose of these experiments at their inception was not to investigate the color reception of chicks, but to test modification of behavior. However, the reactions to reflected colored light proved so interesting that a slight digression from the central interest of the research was later made in the effort to gather some new facts in this closely related field.

Chicks were tried first with Bradley black and white. Most of the first tests involved many elements of irregularity. In some the right and left exchanges of cards were not balanced in the series. In others, chicks had been shocked too severely and either hesitated about entering the electric boxes or balked completely. Again, work was carried over from the preliminary to the new apparatus. The best of these first results are presented below. Chick no. 7, after being given preference trials which were of questionable value, was regularly trained to avoid white and go to black. ${ }^{1}$

TABLE 8

Black-White Reactions

Chick no. 7. Hatched $12 / 10$, , 07 . Sex, F. ${ }^{2}$

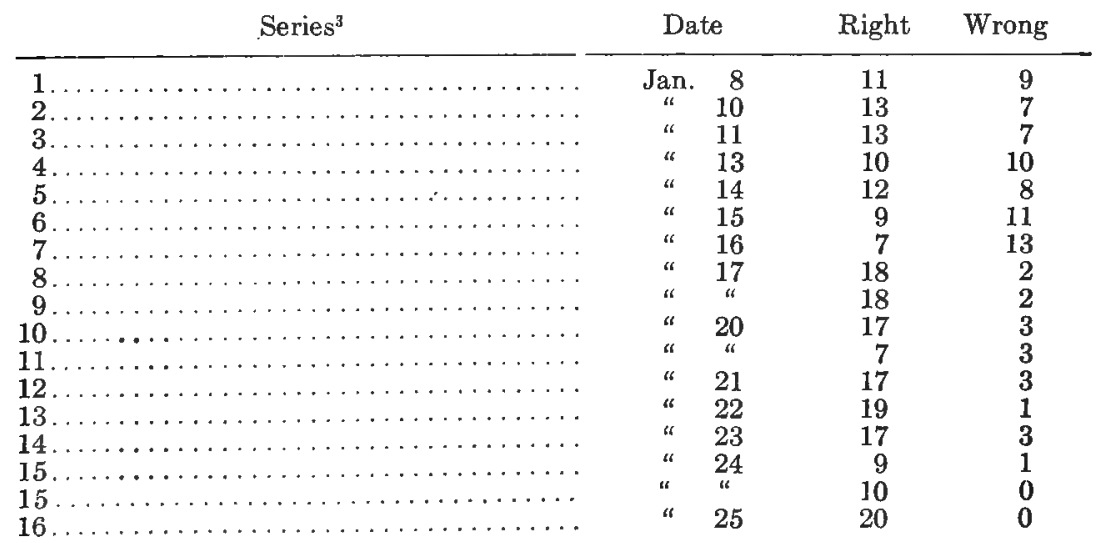

1 In all the tables of results in discrimination work the stimulus to which a chick was forming the habit of positive reaction is each time mentioned first. It is to be assumed that the animals were without previous training unless a definite statement to the contrary is made.

${ }^{2}$ All sex determinations were made by dissection.

3 In some of the preliminary work the regular 10-trial series was doubled. 
In table 9 are presented the results obtained from chick no. 9 under conditions exactly similar to those under which no. 7 was tested. No. 9 , however, was trained to accept white and reject black.

TABLE 9

White-Black Reactions

Chick no. 9. Hatched 12/10, '07. Sex F.

\begin{tabular}{|c|c|c|c|}
\hline Series & Date & Right & Wrong \\
\hline 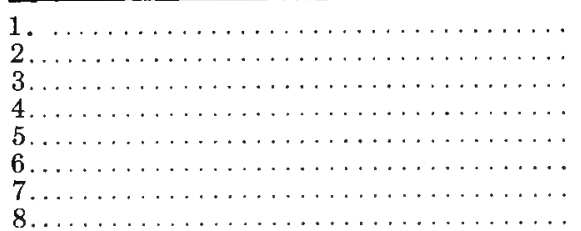 & $\begin{array}{rr}\text { Jan. } & 9 \\
\text { “ } & 10 \\
\text { “ } & 13 \\
\text { “ } & 14 \\
\text { “ } & 15 \\
\text { “ } & 16 \\
\text { “ } & 17\end{array}$ & $\begin{array}{r}11 \\
9 \\
13 \\
12 \\
13 \\
14 \\
20 \\
10\end{array}$ & $\begin{array}{r}9 \\
11 \\
7 \\
8 \\
7 \\
6 \\
0 \\
0\end{array}$ \\
\hline
\end{tabular}

Now, if some animals were going to require, as no. 8,3 Io tests besides preference trials to complete the black-white training, working with this habit would consume too much of the experimenter's time. An objection to the white-black habit lay in the preference prior to training that chicks showed for the white. If a combination of visual stimuli could be discovered for neither of which the chicks showed any marked preference, such a combination would commend itself for use, provided the time required to form the desired habit of reaction should be fairly uniform among different chicks and conveniently short. The combination, orange and white, was tried with the result shown in table Io. The work of chick no. I 2 was devoid of the irregularities spoken of above.

TABLE 10

Orange-White Reactions

Chick no. 12. Hatched $12 / 10$, '07. Sex, F.

Series

Date

Right Wrong

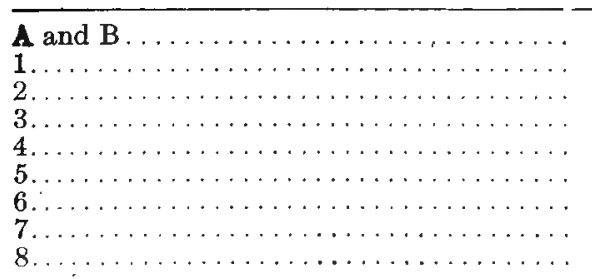

\begin{tabular}{|c|c|c|c|}
\hline Jan. & 29 & 11 & 9 \\
\hline " & 30 & 6 & 4 \\
\hline$"$ & 31 & 6 & 4 \\
\hline Feb. & 1 & 10 & 0 \\
\hline$"$ & 3 & 9 & 1 \\
\hline$"$ & 4 & 9 & 1 \\
\hline$"$ & 5 & 10 & 0 \\
\hline$"$ & 6 & 10 & 0 \\
\hline$"$ & 7 & 10 & 0 \\
\hline
\end{tabular}


This record was the cleanest and most promising so far, but was offset by the results obtained from experiments conducted concurrently upon no. Io. The latter chick, in a period of training twice as long as that given to no. I 2 , failed to acquire a perfect habit of response, to orange. The preference trials in each case were satisfactory.

TABLE 11

Orange-White Reactions

Chick no. 10. Hatched 12/10, '07. Sex, M.

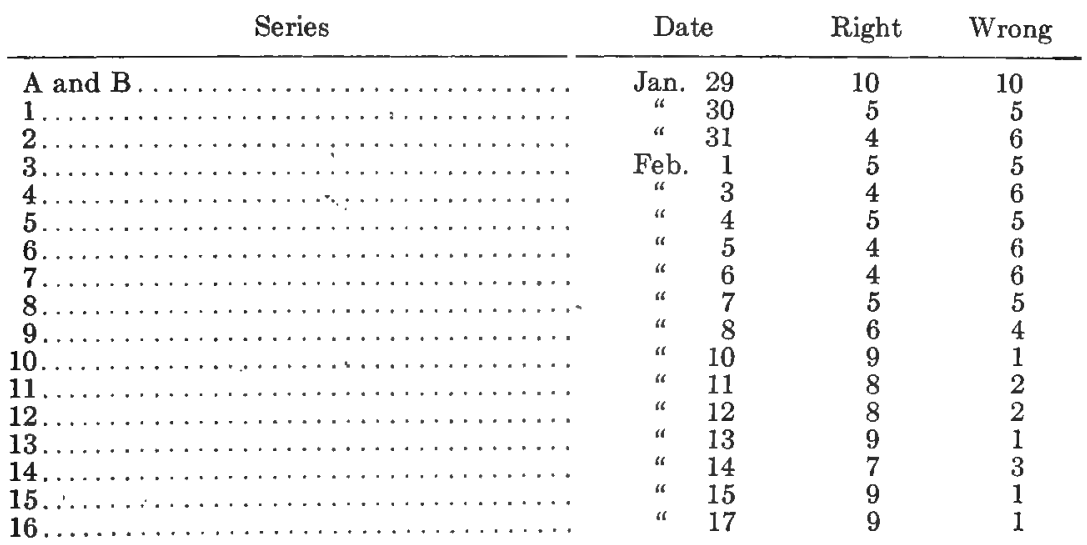

Chick no. 8 was given more searching preference tests and then trained on black-blue.

TABLE 12

Black-Blue Reactions

Chick no. 8. Hatched $12 / 10$, '07. Sex, M.

\begin{tabular}{|c|c|c|c|}
\hline Series & Date & Right & Wrong \\
\hline $\mathbf{A} \ldots \ldots \ldots$ & Jan. 27 & $\begin{array}{c}\text { Orange } \\
13\end{array}$ & $\begin{array}{c}\text { Blue } \\
27\end{array}$ \\
\hline$\ldots \ldots: \ldots \ldots \ldots \ldots \ldots$ & Jan. 28 & $\begin{array}{c}\text { Black } \\
6\end{array}$ & $\begin{array}{c}\text { White } \\
14\end{array}$ \\
\hline $\mathrm{C}, \ldots \ldots \ldots \ldots \ldots \ldots \ldots \ldots \ldots \ldots \ldots \ldots \ldots \ldots$ & Jan. 28 & $\begin{array}{c}\text { Yellow } \\
7\end{array}$ & $\begin{array}{c}\text { Blue } \\
13\end{array}$ \\
\hline$\pi$ & Jan. 29 & $\underset{6}{\text { Black }}$ & $\begin{array}{c}\text { Yellow } \\
14\end{array}$ \\
\hline
\end{tabular}




\begin{tabular}{|c|c|c|c|c|}
\hline & & & Black & Blue \\
\hline$\ldots \ldots$ & Jan. & 29 & 2 & 8 \\
\hline$\ldots \ldots$ & " & 31 & 1 & 9 \\
\hline & Feb. & 1 & 1 & \\
\hline$\ldots \ldots$ & “ & 3 & 2 & \\
\hline$\ldots \ldots \ldots \ldots$ & “ & 4 & 3 & \\
\hline 6. & $"$ & 5 & 6 & \\
\hline …… & $"$ & 6 & 4 & \\
\hline 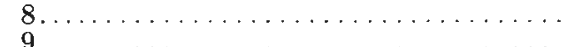 & " & 7 & 8 & \\
\hline 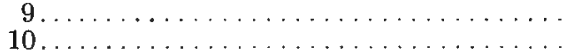 & " & 8 & 7 & \\
\hline 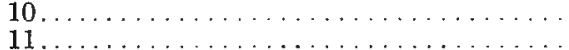 & " & 10 & 8 & \\
\hline$\ldots \ldots \ldots \ldots$ & " & 11 & 8 & \\
\hline 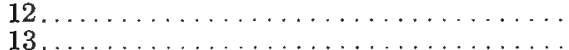 & $"$ & 12 & 10 & \\
\hline 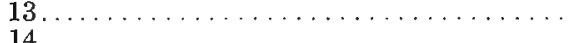 & “ & 13 & 10 & \\
\hline & & 14 & 10 & \\
\hline
\end{tabular}

Preference tests $B$ and $D$, table $\mathrm{I}_{2}$, indicate a natural preference for the brighter of the two cards. The orange-blue and yellow-blue preference tests brought to light interesting data. The yellow here was very light, much brighter than the comparatively dark blue, not only as judged directly by the human eye, but as tested by the flicker method. Particular attention is called to these first reactions to blue for, in so far as this paper has anything to contribute to the study of color vision, the interest will largely center in and results will turn upon the reactions of the chicks to blue.

The training of no. 8 on the black-blue was now carried through with the result detailed in table $\mathrm{x} 2$. Within a reasonable number of trials, I 40, including the final three perfect series, the chick nicely developed the habit of selecting the black. It was thereupon decided to adopt the black-blue combination for a more searching test, with a view to its use in the determination of the rate of modification, the permanence of habit, and some of the effects of training.

Chicks nos. I6, I 7, I8, I9, and 20 were selected for this work. From all appearances they were normal chicks, and were chosen at random from the healthy specimens in a flock of seventeen. They were twelve days old when given their preference tests. In all tests prior to those reported in table $\mathrm{I} 3$ but one set of cards was used, the cards at D, figure 8 . In this set of experiments, after series 2, an additional pair of cards was placed at $F$, whereby shock and color stimuli were presented simultaneously and not successively only, as in the previous experiments. In table 13 the rate of decrease of error or the rapidity of modification is shown in the column, " average number of 
errors." On the basis of these results with black-blue there seemed to have been developed one habit of response to optical stimuli that would serve both for a measurement of the rate of modification in the chick and, when used along with another method yet to be discovered, as a test of the interrelation of modifications.

TABLE 13

\section{Black-Blue Reactions}

Chicks nos. 16 to 20. Hatched 2/5, '08.

No. 16 No. 17 No. 18 No. 19 No. 20 Av.

Sex, F. Sex, F. Sex, F. Sex, F. Sex, M. No.

\begin{tabular}{|c|c|c|c|c|c|c|c|c|c|c|c|c|c|}
\hline Series & Dat & & $\mathrm{R}$ & W & $\mathbf{R}$ & W & $\mathrm{R}$ & W & $\mathrm{R}$ & W & $\mathrm{R}$ & W & rors \\
\hline A. & Feb. & 17 & 5 & 5 & 5 & 5 & 3 & 7 & 4 & 6 & 5 & 5 & 5.6 \\
\hline 1. & & 18 & 3 & 7 & 3 & $\begin{array}{l}0 \\
7\end{array}$ & $\begin{array}{l}0 \\
1\end{array}$ & 9 & 6 & 4 & 4 & $\begin{array}{l}5 \\
6\end{array}$ & $\begin{array}{l}8.0 \\
6.6\end{array}$ \\
\hline 2 . & “ & 19 & 3 & 7 & 3 & 7 & 5 & 5 & 5 & 5 & 6 & 4 & 5.6 \\
\hline 3 . & " & 20 & 6 & 4 & 7 & 3 & 2 & 8 & 3 & 7 & 6 & 4 & 5.2 \\
\hline 4. & " & 21 & 9 & 1 & 8 & 2 & 8 & 2 & 6 & $\dot{4}$ & 8 & 2 & 2.2 \\
\hline 5. & $"$ & 22 & 7 & 3 & 10 & 0 & 5 & 5 & 6 & 4 & 8 & 2 & 2.8 \\
\hline 6. & “ & 24 & 9 & 1 & 9 & 1 & 9 & 1 & 6 & 4 & 9 & 1 & 1.6 \\
\hline 7. & $"$ & 25 & 6 & 4 & 10 & 0 & 7 & 3 & 10 & 0 & 10 & 0 & 1.4 \\
\hline 8 & " & 26 & 10 & 0 & 10 & 0 & 9 & 1 & 10 & 0 & 10 & 0 & 0.2 \\
\hline 9 & " & 27 & 10 & 0 & 10 & 0 & 10 & 0 & 10 & 0 & 10 & 0 & 0 \\
\hline 10. & " & 28 & 9 & 1 & & & 9 & 1 & & & & & 0.4 \\
\hline 11. & " & 29 & 10 & 0 & & & 10 & 0 & & & & & 0 . \\
\hline 12. & Mar. & I & 10 & 0 & & & 10 & 0 & & & & & 0 . \\
\hline 13. & & 2 & 10 & 0 & & & 10 & 0 & & & & & 0. \\
\hline
\end{tabular}

The persistence of habits and their effects, as well as the bearing of the results upon the problem of color vision, will be discussed separately in a later section of the paper. For the present, interest is confined to certain aspects of the process of modification, and the question as to whether the reaction of the chick is determined by the quality as well as the intensity of the color stimulus is not a matter of special concern. The term color is used simply to denote a certain stimulus, with no implication that the chick's reactions are affected by variations in wave-length of the ether vibrations.

Let us turn now to the search for a second method of measuring modification.

\section{B. Form}

To test the animals' responses to forms, black cards, cut as previous cards, were used in the regular card-holders of the apparatus. The cards bore two-dimensional forms above the openings through which a chick had to pass on its way out of the appar- 
atus. That is, forms were cut out of white paper and pasted midway between the two sides and a little below the top of the card. The chicks were first tested with a square and a triangle of equal area. The triangle had a base of $8.13 \mathrm{~cm}$. and an altitude of $6.35 \mathrm{~cm}$. The square was $5.08 \times 5.08 \mathrm{~cm}$. No. II, a chick that had succeeded in acquiring the black-white habit to the point of making one error in the last twenty trials, reacted in the square-triangle test as shown in table $\mathrm{I} 4$.

TABLE 14

Reactions to Form

Chick no. 11. Hatched 12/10, '07. Sex, F.

Square-Triangle

\begin{tabular}{|c|c|c|c|}
\hline Series & Date & Right & Wrong \\
\hline A & Jan. 31 & 5 & 5 \\
\hline 1. & " " " & 1 & 9 \\
\hline$\ldots$ & Feb. 1 & 3 & 7 \\
\hline$\ldots \ldots \ldots$ & " 3 & 4 & 6 \\
\hline$\ldots \ldots \ldots \ldots$ & “ 4 & 6 & 4 \\
\hline$\ldots \ldots \ldots \ldots \ldots \ldots$ & " 5 & 6 & 4 \\
\hline$\ldots \ldots$ & " 6 & 4 & 6 \\
\hline$\ldots \ldots \ldots$ & 7 & 7 & 3 \\
\hline$\ldots \ldots$ & 8 & 5 & 5 \\
\hline$\ldots \ldots$ & $" 10$ & 2 & 8 \\
\hline$\ldots$. & " 11 & 4 & 6 \\
\hline$\ldots \ldots \ldots \ldots \ldots \ldots$ & " 12 & 6 & 4 \\
\hline$\ldots \ldots \ldots \ldots$ & “ 13 & 2 & 8 \\
\hline$\ldots \ldots \ldots$ & “ 14 & 3 & 7 \\
\hline$\ldots \ldots \ldots$ & “ 15 & 6 & 4 \\
\hline 15. & “ 17 & 4 & 6 \\
\hline
\end{tabular}

Not only were the results here purely negative, but one had only to watch the chick to become convinced that the probability of its forming the habit of selecting the square was almost nil.

No. I 3 , a chick that had acquired the white-black (darkened ${ }^{1}$ ) habit perfectly, was tried at the same time by the same method in this form test with the result shown in table 15 .

No. I 3 showed no signs of improvement and the experiment was discontinued. The square-triangle combination was discarded for a "design" that was placed at the top of the cards. This time the cards in the apparatus were white and the forms upon them black. On one set of cards were pasted, vertically

${ }^{1}$ The electric chamber on the side of black was covered with cardboard. 
and $2.5 \mathrm{~cm}$. apart, two parallel bands. of black paper, each band being rectangular in form and measuring $4.2 \mathrm{x} .9 \mathrm{~cm}$. On the other set of cards two black bands of equal area and the same material were fastened at right angles to each other and in this wise $(\Lambda)$. Chick no. 2 I was tried first with this combination of forms, but with negative results. No. I 5 was tested at the same time. It showed no improvement after 60 trials and from that on the continuous training plan was used. On Mar. I it was given I60 trials. The regular system of card exchanges was disregarded and one setting of cards was allowed to remain during several successive trials to see if the chick might thus hit upon the habit of selecting the vertical bars. Sixty trials were given in a similar way on Mar. 2 and as many more on Mar. 3, with negative results.

TABLE 15

Reactions To Form

Chick no. 13. Hatched 12/10, '07. Sex, F.

Triangle-Square

\begin{tabular}{|c|c|c|c|}
\hline Series & Date & Right & Wrong \\
\hline 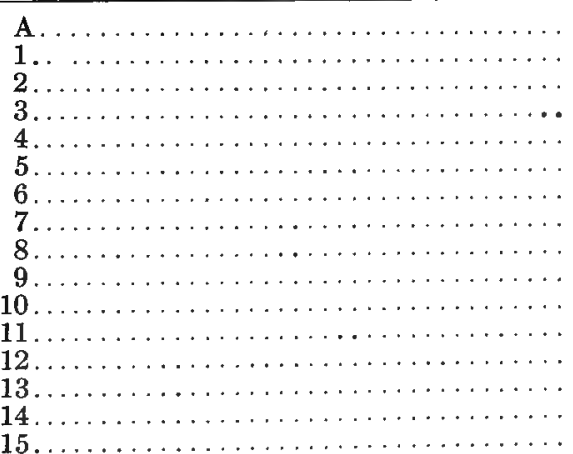 & $\begin{array}{cr}\text { Jan. } & 30 \\
\text { “ } & 31 \\
\text { Feb. } & 1 \\
\text { " } & 3 \\
\text { " } & 4 \\
\text { " } & 5 \\
\text { " } & 6 \\
\text { " } & 7 \\
\text { " } & 8 \\
\text { " } & 10 \\
\text { " } & 11 \\
\text { " } & 12 \\
\text { " } & 14 \\
\text { " } & 15 \\
\text { " } & 17\end{array}$ & $\begin{array}{l}5 \\
3 \\
4 \\
8 \\
5 \\
6 \\
3 \\
5 \\
4 \\
6 \\
7 \\
2 \\
5 \\
7 \\
6 \\
5\end{array}$ & $\begin{array}{l}5 \\
7 \\
6 \\
2 \\
5 \\
4 \\
7 \\
5 \\
6 \\
4 \\
3 \\
8 \\
5 \\
3 \\
4 \\
5\end{array}$ \\
\hline
\end{tabular}

The plan of having forms at the tops of the display cards was then abandoned and another scheme employed. This time display cards were made of gray cardboard and the forms were presented as openings in the cards through which the chick had to pass on its way out of the apparatus. In one set of cards a circular opening was cut the diameter of which was $8.47+\mathrm{cm}$. In the other set of cards a rectangular opening was cut whose base was $6.35 \mathrm{~cm}$. and altitude 8.89 . The circle and 
the rectangle were constructed as nearly as possible of equal areas. The regular manner of training was resumed and the reactions of chicks I 5,22 , and 23 to the circle-rectangle combination were tested. At the risk of being tedious I present the results in detail, for whether or not the ability to react selectively in this case would have demonstrated the dependence of the reactions on the element of form, it did not seem unreasonable to expect the chick to develop a habit of reacting differently to stimuli as different as these.

A glance at tables $\mathrm{i} 6, \mathrm{I} 7$, and 18 reveals only negative results. The chicks soon came to a point in these form tests where they did not make an effort to escape from the box. Precaution was taken to see that the electric shock was not too severe. Previous experience in the color experiments had shown that a chick occasionally received a shock of too great intensity and thereafter hesitated about entering the electric chambers. But the shock for nos. 15 and 23 ran as low as 6 to 6.5 on the scale of the inductorium, and did not reach a greater intensity than 6.5 for no. 22. The regular shock, as stated before, was 5.5. Just prior to the discontinuance of the tests, no. 22 would approach the display cards, hesitate, at times shake its bill, and go no further. The expedients of moving a card over the top of the apparatus from the entrance box toward the

TABLE 16

Reactions to Form

Chick no. 15. Hatched 2/5, '08. Sex, Undetermined

Circle-Square

\begin{tabular}{|c|c|c|c|c|}
\hline Series & Dat & & Right & Wrong \\
\hline$\ldots \ldots \ldots$ & Mar. & 6 & 4 & 6 \\
\hline$\ldots \ldots \ldots \ldots \ldots \ldots \ldots \ldots$ & " & $"$ & 2 & 8 \\
\hline 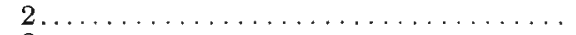 & “ & " & 2 & 8 \\
\hline$\ldots \ldots$ & " & $"$ & $\overline{3}$ & 7 \\
\hline$\ldots$ & “ & " & 4 & 6 \\
\hline$\ldots \ldots$ & “ & 7 & 4 & 6 \\
\hline .. & “ & $"$ & 4 & 6 \\
\hline$\ldots \ldots$ & “ & " & 2 & 8 \\
\hline$\cdots$ & “ & 8 & 2 & 8 \\
\hline$\ldots \ldots$ & “ & " & 4 & 6 \\
\hline$\ldots$ & “ & " & 6 & 4 \\
\hline$\ldots \ldots \ldots \ldots \ldots \ldots \ldots \ldots$ & $"$ & $"$ & 5 & 5 \\
\hline$\ldots \ldots \ldots$ & “ & 9 & 5 & 5 \\
\hline 13. & ." & “ & 4 & 6 \\
\hline 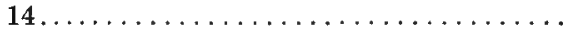 & $"$ & 10 & 3 & 7 \\
\hline
\end{tabular}


TABLE 17

REACTIONS TO FORM

Chick no. 22. Hatched 2/5, '08. Sex, F.

Circle-Square

\begin{tabular}{|c|c|c|c|}
\hline Series & Date & Right & Wrong \\
\hline 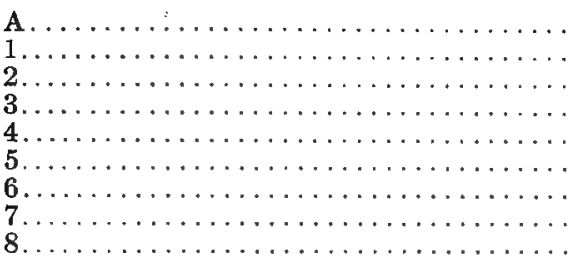 & $\begin{array}{cc}\text { Mar. } & 9 \\
\text { " } & 10 \\
\text { " } & 12 \\
\text { " } & 12 \\
\text { " } & 13 \\
\text { " } & \text { “ } \\
\text { " } & 16\end{array}$ & $\begin{array}{l}4 \\
4 \\
5 \\
5 \\
5 \\
4 \\
2 \\
6 \\
4\end{array}$ & $\begin{array}{l}6 \\
6 \\
5 \\
5 \\
5 \\
6 \\
8 \\
4 \\
6\end{array}$ \\
\hline
\end{tabular}

TABLE 18

Reactions to Form

Chick no. 23. Hatched 2/5, '08. Sex, Undetermined

Circle-Square

\begin{tabular}{|c|c|c|c|}
\hline Series & Date & Right & Wrong \\
\hline A..... & $\underset{u}{\operatorname{Mar} .} \underset{u}{9}$ & 4 & 6 \\
\hline$\ldots \ldots \ldots \ldots \ldots \ldots$ & " & 9 & 1 \\
\hline $\begin{array}{l}(\cdots \\
1\end{array}$ & " 10 & 2 & 8 \\
\hline & “ 12 & $\begin{array}{l}0 \\
2\end{array}$ & $\begin{array}{l}4 \\
8\end{array}$ \\
\hline$\therefore \ldots \ldots \ldots \ldots$ & 13 & 6 & 4 \\
\hline$\ldots \ldots, \ldots \ldots \ldots$ & “ & 6 & 4 \\
\hline$\cdots$ & 16 & 8 & 2 \\
\hline$\ldots \ldots \ldots \ldots$ & 17 & 5 & 5 \\
\hline . . & 19 & 4 & 6 \\
\hline & 20 & 5 & 5 \\
\hline
\end{tabular}

chick and of forcing him gently from behind were tried without effect. The chick simply "gave up," as my note has it. ${ }^{1}$

\section{Size}

The search for a second method of measuring modification of behavior was continued in an investigation of the reactions of the chick to size differences in two dimensions, difference in form being excluded. The plan was adopted of having openings in gray display-cards through which the chick had to pass. The openings in one set of cards were $6.35 \mathrm{~cm}$. wide and $8.89 \mathrm{~cm}$. high; those in the other set $8.89 \mathrm{~cm}$. wide and $12.44 \mathrm{~cm}$. high.

${ }^{1}$ Later, during some work as yet unpublished, I found a chick that acquired the circle-square habit when the experiment was conducted in a dark-room. Opal glass was electrically lighted from behind and the above forms, cut in black tin screens, permitted illuminated areas of these shapes to be exposed to the chick. 
The openings were the same in shape and one was approximately twice the size of the other. The experiment was undertaken with chicks no. 24 and no. 25 , and completed with the result detailed in table r 9 . The training was finished quite satis-

TABLE 19

Reactions to Size: Small-Large Openings

Chicks nos. 24 and 25. Hatched 2/5, '08

\begin{tabular}{|c|c|c|c|c|c|}
\hline \multirow[b]{2}{*}{ Series } & \multirow[b]{2}{*}{ Date } & \multicolumn{2}{|c|}{$\begin{array}{l}\text { No. } 24 \\
\text { Sex, M. }\end{array}$} & \multicolumn{2}{|l|}{$\begin{array}{l}\text { No. } 25 \\
\text { Sex, F. }\end{array}$} \\
\hline & & $\mathrm{R}$ & W & $\mathbf{R}$ & W \\
\hline A. & Mar. 17 & 5 & 5 & 3 & 7 \\
\hline$\cdots$ & " 18 & 0 & 10 & 3 & 7 \\
\hline 2 . & “" " & 3 & 7 & (frightened & 1?) \\
\hline$\ldots \ldots$ & “ 19 & 5 & 5 & 4 & 6 \\
\hline$\ldots \ldots$ & " " & 8 & 2 & 6 & 4 \\
\hline . & “ 20 & 7 & 3 & 7 & 3 \\
\hline $6 \ldots \ldots \ldots \ldots \ldots \ldots$ & " " " & 7 & 3 & 5 & 5 \\
\hline$\ldots$ & “ 21 & 8 & 2 & 3 & 7 \\
\hline$\ldots \ldots$ & " & 10 & $\overline{0}$ & 6 & 4 \\
\hline$\ldots \ldots \ldots$ & “ 23 & 10 & 0 & 10 & 0 \\
\hline$\ldots \ldots \ldots \ldots \ldots$ & “ & 10 & 0 & 10 & 0 \\
\hline & 25 & & & 9 & 1 \\
\hline
\end{tabular}

Control Tests

Card of small opening brighter, and its electric box brighter:

$1 \ldots \ldots \ldots \ldots \ldots \ldots \ldots \ldots \ldots \ldots$ Mar. $24 \quad 9 . \ldots 1$

Card of large opening brighter, its electric box brighter:

$2 \ldots \ldots \ldots \ldots \ldots \ldots \ldots \ldots \ldots \ldots \ldots$ Mar. $24 \quad 9 . \ldots 1$

Cards equally illuminated, electric box of small opening made the brighter by illumination of electric light:

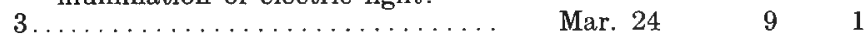

Card of small opening brighter, electric box of large opening made much darker by overhead cardboard:

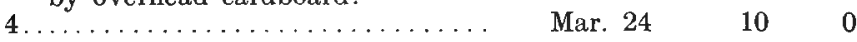

Cards equally illuminated, electric box of large opening made the darker by overhead cardboard:

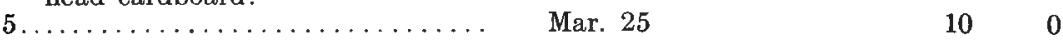

factorily and within a reasonable time limit, so the method seemed available for further use. Five control series were given to check out the influence of brightness. It is manifest that with equal illumination on the faces of the display-cards, which was roughly secured by adjustment of the shades of the two windows that admitted light to the room, the electric box back 
of the large opening would be brighter than that back of the small one. The angle at which the cards stood to each other, together with the position of the windows with reference to the cards, made it possible to vary the brightness of the two cards independently, and that of the electric boxes so that the electric box on the side of the brighter card could be made also the brighter box. A cardboard over an electric box was also used to screen off light. Within the degrees of variation employed, the chicks continued to select the smaller opening regardless of the relative brightness of the faces of the cards or the electric boxes. When both electric chambers were covered with a ground glass plate and a 2 c.p. electric light was adjusted over the electric box on the side of the small opening, no. 24 approached as usual the small opening, hesitated a moment, glanced about the apparatus in the vicinity of the card, and then slowly entered. (See control test 3, table I 9.) Control 5 exhibits the same general relations as 3 , but without the use of electric light. The pronounced variation, even to a different quality of light, did not inhibit the selective reaction. In control series 4 the electric box on the side of the card having the large opening was darkened considerably by cutting off the light from above with a piece of black cardboard. The figures for this series of reactions, I0-O, in comparison with the $9-\mathrm{I}$ records in the other series, represent but do not express fully the difference in readiness with which the chick responded. This series repeated control series I with greater difference in the relative brightness of the electric boxes. The conclusion seems to be justified, on the basis of these results, that the chicks were responding selectively to one of two objects of different size. Of course the control of other possible determinants of reaction, such as odor, would have made this conclusion more certain. The small-large habit was now adopted to be used in conjunction with the black-blue habit for an experimental test of the effects and interrelation of habits.

\section{To What Extent Has Training General Value?}

After an experimental test of the influence of one labyrinth habit upon the formation of another, Yerkes ${ }^{2}$ concludes that "the acquisition of one form of labyrinth habit may facilitate

1 Yerkes, R. M.: Op. cit., p. 261. 
the acquisition of others." "For the student of animal behavior, as for the human educator, it is of importance to learn," he writes, "whether one kind of training increases the efficiency of similar forms of training." The question for which an answer was sought in the work about to be reported is closely related to the problem which Yerkes set for himself, but is concerned with the more general value of training: How does acquired ability to react perfectly to one kind of element or complex affect the acquisition of ability to react to a quite different element or complex?

Among certain organisms a particular act once acquired is more easily re-acquired. A particular act once acquired may facilitate the acquisition of a similar act, similar meaning here a relation of partial identity. Now the question naturally arises, As two acts are less and less similar, what effect does the acquisition of one have on the later acquisition of the other? This raises the problem of formal discipline, capable, surely, of solution by experiment, but not yet solved. It has been suggested that there may be "two kinds or aspects of organic modification in connection with training; those which constitute the basis of a definite form of motor activity, and those which constitute the bases or dispositions for the acquirement of certain types of behavior." " The data upon which this suggestion is based would allow us to say nothing definite about "the acquirement of certain types of behavior" not of the original labyrinth form, which Yerkes used. But at that the general value of training has been experimentally demonstrated, in so far as different labyrinths are different objects, even different objects of the same kind. Although there is a degree of generality here, it is so slight that the training value involved would no doubt ordinarily be classified as specific. If no two particular stimuli are identical, then, practically speaking, much specific training has general value, which does not mean, however, that this generality of value may not rest at bottom on specific modifications. This must be our conclusion when we look at the matter from the side of content as opposed to function, stimulus as opposed to reaction. Practically, it cannot be denied that in some organisms certain kinds of training do have value for certain other kinds. The question awaiting answer in the

${ }^{1}$ Yerkes, R. M.: Op. cit., p. 261. 
present state of our knowledge is, Between what kinds of modifiactions and to what degree is there transfer? Few will dispute the assertion that there may be dispositions preceding and underlying the acquisition of a particular act which are not identical with those that are at the basis of its actual performance. Indeed the former may no doubt be congenitally present, with or without the actual ability to perform. In a similar manner, of course, one may think of an acquired neurological disposition "predisposing" the individual to certain new activities. And within what range? That is our problem.

To bring a particular case to a test, two comparable groups of four chicks each were chosen, Group I consisting of chicks nos. $32,33,38$, and 39 , Group II of chicks nos. $34,35,36$, and 40. All the chicks were of the same brood, hatched April I3, I go8. None of them had any other training prior to or during the course of these experiments. Uniformity in method was strictly adhered to. The chicks of Group I were given the black-blue training to the point where they made no errors for three successive days, ten trials per day. Then the chicks of both grours entered together into the size training. During the course of the development of each habit one setting of the cards was used for the corresponding trial of all the animals. For example, in the black-blue work, the black card occupied the positions $r, 1, r, 1, r, r, 1,1, r, 1$, in each series until an errorless series was reached. At the first setting of the cards-black right, blue left-the chicks of Group I went through one at a time until each animal had to its credit one reaction. Then the cards were exchanged to the second position and the chicks again taken as before.

As a precaution which seemed advisable, the secondary coil of the inductorium was adjusted at 6 to begin with in the blackblue training, and it was continued thus throughout. In the later size training a shock of the same intensity was employed at the start and at the beginning of the ninth series this was raised to 5.5 on all the chicks alike.

The order of training was this. When the chicks of Group I were twelve days old, work was begun with them, and they were trained until they responded errorlessly with a positive reaction to black. On May II, by which time this group had finished the color work, these animals with those of Group II 
were introduced into the size training. Table 20 shows the course of modification of response to the color stimuli. Only the errors appear in this summary.

TABLE 20

Errors in Black-Blue Reactions

Chicks nos. $32,33,38$, And 39 . Hatched $4 / 13$, '08

Group I.............. $32 \quad 33 \quad 38 \quad 39$

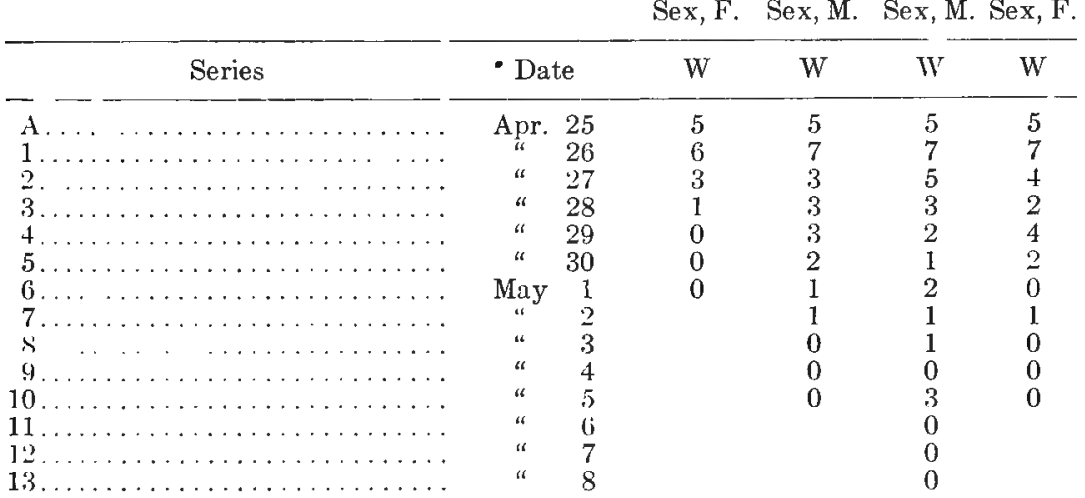

These chicks really do not vary so much in the modifiability of their behavior as the figures might indicate. No. 38 became excited during the tenth series, which may explain its lapse. The rapidity with which no. 32 finished the work is noticeable. No chick before or afterwards in my experiments excelled this record of 30 trials exclusive of preference and perfect series. But it is sufficient for our present purpose simply to show clearly the perfection of the training that preceded the formation of the second habit, without extended discussion of the other characteristics of the modification.

Following the completion of this training of Group I, both groups were introduced into a size experiment, as described above. Table 2 I reveals the progressive development of the second modification for each group in the column of average daily number of errors. The total of these averages for Group I amounts to 66.3 for the sixteen days training; for Group II, 70.I during the same period. Curves I and II, fig. IO, exhibit graphically the rate of decrease of error for the corresponding groups. The record of each group in the preference tests is 
included in the curves. In a comparison of results for the two groups two things especially must be taken into consideration, (I) the natural preferences before training began and (2) previous training. In regard to the first, Group II had to overcome a stronger preference for the larger of the two openings. The chicks of Group I took the smaller opening more often than those of Group II, perhaps because in general the black-blue

TABLE 21

RECORD OF ERRORS

Reactions to Size: Smali-Large Openings

HATChED 4/13, '08

\begin{tabular}{|c|c|c|c|c|c|c|c|c|c|c|c|c|}
\hline & & & & & roup & & & & & oup & & \\
\hline Series & Dat & & 32 & 33 & 38 & 39 & Av. & 34 & 35 & 36 & 40 & Av. \\
\hline A... & May & 11 & 6 & 7 & 5 & 3 & 5.3 & 5 & 5 & 7 & 8 & 6.3 \\
\hline $1 \ldots \ldots \ldots$ & & 12 & 8 & 5 & 9 & 7 & 7.3 & 8 & 9 & 5 & 9 & 7 \\
\hline$\ldots \ldots$ & “ & 13 & 6 & 7 & 6 & 7 & 6.5 & 6 & 9 & 10 & 5 & 7 \\
\hline$\ldots \ldots$ & “ & 14 & 6 & 5 & 6 & 8 & 6.3 & 6 & 7 & 5 & 7 & 6.3 \\
\hline$\ldots$ & “ & 15 & 7 & 7 & 4 & 7 & 6.3 & 5 & 5 & 7 & 7 & 6 \\
\hline 5. & & 16 & 5 & 5 & 5 & 7 & 5.5 & 7 & 8 & 6 & 7 & 7. \\
\hline 6 . & & 18 & 5 & 4 & 6 & 3 & 4.5 & 6 & 6 & 4 & 6 & 5.5 \\
\hline & & 19 & 7 & 7 & 2 & 1 & 4.3 & 3 & 4 & 3 & 6 & \\
\hline . & & 20 & 5 & 8 & 2 & 3 & 4.5 & 2 & 6 & 1 & 5 & 3 \\
\hline 9 . & “ & 21 & 3 & 5 & 4 & 2 & 3.5 & 5 & 4 & 1 & 8 & \\
\hline 10 . & & 22 & 4 & 7 & 3 & 2 & 4. & 3 & 3 & 3 & 6 & 3 \\
\hline 11. & “ & 23 & 6 & 3 & 2 & 2 & 3.3 & 1 & 2 & 2 & 6 & \\
\hline 12 . & “ & 25 & 4 & 6 & 2 & 4 & 4 & 2 & 3 & 0 & 4 & 2.3 \\
\hline$\ldots \ldots \ldots$ & “ & 26 & 1 & 1 & 1 & 1 & 1 & 1 & 2 & 4 & 5 & \\
\hline$\ldots \ldots$ & " & 27 & 1 & 2 & 1 & 2 & 1 . & 1. & 2 & 3 & 4 & 2 \\
\hline$\ldots \ldots$ & " & 28 & 0 & 1 & 4 & 1 & 1.3 & 1 & 1 & 1 & 4 & 1.8 \\
\hline 16. & " & 29 & 2 & 1 & 5 & 1 & 2.3 & 1 & 0 & 1 & 5 & 1.8 \\
\hline
\end{tabular}

training inclined chicks to accept the stimulus of lower brightness value, which in this case has been shown to be on the side of the smaller opening. The previous brightness training here came into conflict, however, with the natural(?) tendency to pass out by the larger opening, which may be something more than a brightness preference, and thus may be explained the small difference between the two groups in the results of the preference trials. As to the second point above, Group I, besides acquiring the black-blue habit, had become habituated to the general conditions of the apparatus in such a way that adjustment to the new conditions of the size experiment must have 
been somewhat facilitated-through a lessening of the tendency to general excitement during the first tests, if in no other way. In view of these considerations there is no conclusive evidence that the previous training of Group I with black-blue facilitated the formation of the small-large habit, albeit Group I averaged $5.4 \%$ less errors per day than Group II.

It should be stated in connection with the above size work that the results indicate difficulty of acquisition. The behavior of the chicks pointed in the same direction. So, any more general conclusion than we have ventured would have to be made sub-

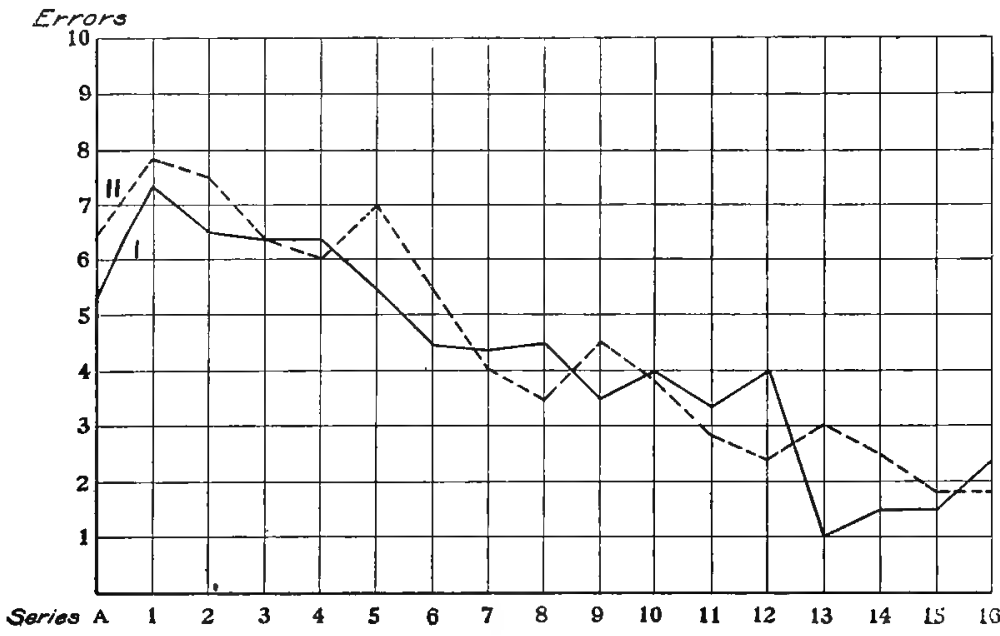

Figure 10-Rate of modification in size training, arranged to test the effect of previous color training. Curve I shows the rate of acquisition by Group I of the small-large habit after having acquired the black-blue habit; Curve II represents the rate of acquisition of the same habit by Group II, the same number and the same age as the chicks in Group I, but without previous color training. Preference tests on first day. Distances along axis of abscissae represent series; along axis of ordinates, a verage number of errors.

ject to this further fact. It ought also to be pointed out that chicks of the same age are not always of the same size. The members within each group were of unequal size when six weeks old. Yet, fortunately for our experiment, the groups remained comparable as to size, individual for individual, throughout the tests. This matter is vital to the experiment, for naturally chicks of different sizes could pass through the small opening with different degrees of ease. 
But the conclusion as to the general efficiency of the blackblue training should not rest alone upon a comparison of the rates of modification in the two groups of chicks. Having learned in the course of the experimentation with the blackblue habit, from data which will appear later, that this modification persists for some time with relatively slight change, it seemed of value to investigate the relation of the size training to the color training by means of the so-called memory test.

TABLE 22

The Non-interference of Modifications

\begin{tabular}{|c|c|c|c|c|c|c|c|c|c|c|}
\hline \multirow[b]{3}{*}{ No. } & \multicolumn{4}{|c|}{$\begin{array}{l}\text { Training in } \\
\text { Black-Blue }\end{array}$} & \multicolumn{3}{|c|}{$\begin{array}{l}\text { Training in } \\
\text { Small-Large }\end{array}$} & \multicolumn{3}{|c|}{$\begin{array}{l}\text { Persistence of } \\
\text { the Black-Blue } \\
\text { Modification }\end{array}$} \\
\hline & \multirow[b]{2}{*}{ Age } & \multirow[b]{2}{*}{ Sex } & \multirow[b]{2}{*}{ Trials } & \multirow[b]{2}{*}{ Errors } & \multirow[b]{2}{*}{ Age } & \multirow[b]{2}{*}{ Trials } & \multirow[b]{2}{*}{ Errors } & \multirow{2}{*}{$\begin{array}{l}\text { Inter- } \\
\text { val }\end{array}$} & \multirow[b]{2}{*}{$\mathbf{R}$} & \multirow{2}{*}{$\begin{array}{l}\text { First } \\
\text { selec- } \\
W \text { tion }\end{array}$} \\
\hline & & & & & & & & & & \\
\hline $\begin{array}{l}32 \\
33 \\
38 \\
39\end{array}$ & $\begin{array}{l}12 \\
12 \\
12 \\
12\end{array}$ & $\begin{array}{l}\mathrm{F} \\
\mathrm{M} \\
\mathrm{M} \\
\mathrm{F}\end{array}$ & $\begin{array}{r}30 \\
70 \\
100 \\
70\end{array}$ & $\begin{array}{l}10 \\
20 \\
25 \\
20\end{array}$ & $\begin{array}{l}28 \\
28 \\
28 \\
28\end{array}$ & $\begin{array}{l}160 \\
160 \\
160 \\
160\end{array}$ & $\begin{array}{l}70 \\
74 \\
62 \\
58\end{array}$ & $\begin{array}{l}32 \\
30 \\
31 \\
30\end{array}$ & $\begin{array}{r}10 \\
10 \\
4 \\
9\end{array}$ & $\begin{array}{ll}0 & \text { Black } \\
0 & \text { Black } \\
6 & \text { Blue } \\
1 & \text { Blue }\end{array}$ \\
\hline
\end{tabular}

Table 22 exhibits the results of this test. The table shows the age of the chicks of Group I when the work on black-blue was begun, I 2 days; the number of trials necessary to complete the training, exclusive of preference and final perfect series; the number of errors made in the course of this training; and also the corresponding age, trials and errors for the size training. The interval, thirty days or more in each case, is the number of days between the date when the black-blue modification was pronounced perfect and the date upon which the memory test was given. Of course sixteen days of this interval were occupied with the development of the small-large habit.

The reactions of the chicks to black-blue in the persistence tests are detailed in the columns $\mathrm{R}$ and $\mathrm{W}$. Chicks nos. 32 and 33 made perfect records, no. 39 made an error on its first trial, being thereafter perfect, while the reactions of no. $3^{8}$ seemed to show no trace of the original modification. The results altogether weigh in favor of the view that the modifications with which we have dealt proceed quite independently 
of each other. By consulting table 26 it will be seen that the persistence of the black-blue habit in these chicks after 30 days measures fully up to the average made by other chicks that had not had intervening training. If it be objected at this point that one should expect the small-large training to exert an influence in the direction of strengthening the black-blue modifcation, I agree, in so far as brightness is concerned, but the inference is that something more than brightness was involved in each habit. That color and not brightness was the effective element in these tests of persistence I have not shown. The results are offered in their present stage for their possible value as a suggestion for method. It is not too much to expect relative permanency of the black-blue habit even if the brightness relations of the first training were reversed in the second. Though both forms of training are habits of response to optical stimuli, they are probably quite separate from each other in their development. Physiologically, this likely means the formation of separate neural bases. For our explanations we look to the central nervous system. Judd" observes that "Discrimination is not a process of impression." "The raw materials for adaptation, the impressions, are there very early, waiting for the individual gradually to adjust himself to them." Discrimination depends upon the ability of the individual to react specifically. Capacity to discriminate is linked with the capacity to react. If this be true, the animal's scope of reaction will determine the limits of its modifiability. According to this view, the grouping of elements to form a single object of reference in reaction derives its unity from the co-ordination in a single active brain process. This does not ignore the fact that the structure of the end organs will also define in advance the range of objects that may be discriminated. Impressions to be distinct must be accompanied by distinctive netrological dispositions. "In short," says Judd again, "so far as this raw material for development which presents itself in the form of impressions is concerned, it must all be worked over and connected with individual reactions before it can be regarded as really assimilated by the developing individual."

But let us return from theory to facts.

${ }^{1}$ Judd, C. H.: Genetic psychology for teachers. New York, 1903, p. $153 \mathrm{ff}$. 
E. Some Effects of Modifications

In table $\mathrm{I} 2$ were pointed out the natural color preferences of chick no. 8: white in preference to black, blue to orange, blue to yellow, and yellow to black. The peculiarity of the reactions to blue was mentioned. If blue be supposed to have a higher brightness value for chicks than for humans, the chick's behavior might be satisfactorily explained on the ground only of brightness of stimulus. Further experimentation was undertaken with a view to an explanation of the above apparent irregularity. No. I , a chick which had been trained on blackwhite and had progressed so far that in its last series of twenty successive trials it made but one error, was tested for its preference on the combination of colors listed in table 23. Fight days had elapsed between the date of the I9-I series and these color preference tests.

TABLE 23

Preference Tegts After Black-White Training

Chick no. 11. Hatched 12/10, '07. Sex, F.

\begin{tabular}{|c|c|c|c|}
\hline Series & Date & Right & Wrong \\
\hline & Jan. 21 & $\begin{array}{c}\text { Black } \\
19\end{array}$ & $\begin{array}{c}\text { White } \\
1\end{array}$ \\
\hline A.... & Jan. 29 & $\underset{8}{\text { Orange }}$ & $\begin{array}{c}\text { White } \\
2\end{array}$ \\
\hline B. & Jan. 29 & $\begin{array}{c}\text { Black } \\
10\end{array}$ & $\begin{array}{c}\text { Blue } \\
0\end{array}$ \\
\hline C . ... & Jan. 29 & $\begin{array}{c}\text { Blue } \\
6\end{array}$ & $\begin{array}{c}\text { White } \\
4\end{array}$ \\
\hline D. & Jan. 29 & $\begin{array}{c}\text { Yellow } \\
8\end{array}$ & $\begin{array}{c}\text { White } \\
2\end{array}$ \\
\hline
\end{tabular}

This record reports the tests in the order in which they were given, as do other records. Again the reactions to blue seemed somewhat surprising. That blue should be rejected as freely as white, when appearing with black, and that blue and white should be accepted almost indifferently, are not results that one should expect. These facts seem to confirm the hypothesis that blue has a special brightness value for the chick.

Further investigation followed with six other chicks, incidental to the direct line of investigation. Table 24 shows the first preference reactions of chicks nos. I7, 18, 20, 32, 33, and 
39 to black-blue. The preference tests are not representative of our chicks in general, for those chicks that already had a preference for black were rejected because they were not suitable for the special purposes of the experiment of which this blackblue training formed a part. There were a few of these. But this does not affect in the least the point that I wish to make in connection with the effects of training. The state in which the black-blue modification persisted at the time of the later color preference tests is shown in the columns under "Persistence." The tests on blue-white, black-white, blue-yellow,

TABLE 24

Some Effects of Tratning

\begin{tabular}{|c|c|c|c|c|c|c|c|c|c|c|c|c|c|c|c|c|c|c|c|}
\hline \multirow[b]{2}{*}{ 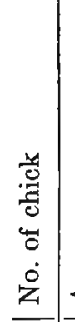 } & \multirow[b]{2}{*}{ 嵒 } & \multirow[b]{2}{*}{ 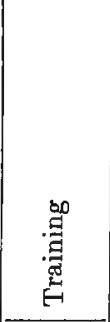 } & \multicolumn{2}{|c|}{$\begin{array}{c}\begin{array}{c}\text { Prefer- } \\
\text { ence }\end{array} \\
\end{array}$} & \multicolumn{4}{|c|}{$\begin{array}{c}\text { Persist- } \\
\text { ence }\end{array}$} & \multicolumn{11}{|c|}{ Preference after Training } \\
\hline & & & 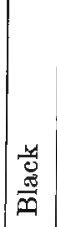 & $\frac{g}{2}$ & 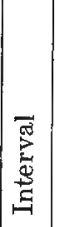 & $\left|\begin{array}{l}\frac{m}{0} \\
\ddot{m} \\
\ddot{m}\end{array}\right|$ & $\stackrel{\Phi}{\underline{\Xi}}$ & 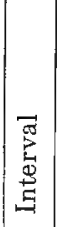 & $\stackrel{\Phi}{\vec{m}}$ & 总 & $\mid \begin{array}{l}m \\
\frac{m}{0} \\
\underline{m}\end{array}$ & $\mid$ & 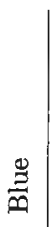 & $\begin{array}{l}0 \\
0 \\
0 \\
0\end{array}$ & 焉 & 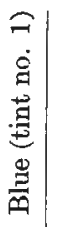 & 总 & 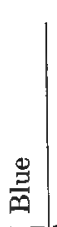 & 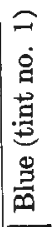 \\
\hline $\begin{array}{l}17 \\
18\end{array}$ & $\begin{array}{l}12 \\
12 \\
12 \\
12 \\
12 \\
12 \\
39 \\
39\end{array}$ & \begin{tabular}{|c} 
Black- \\
Blue \\
$a$ \\
$“$ \\
$“$ \\
None \\
“
\end{tabular} & $\begin{array}{l}5 \\
3 \\
5 \\
5 \\
5 \\
5 \\
1 \\
1\end{array}$ & $\begin{array}{l}5 \\
7 \\
5 \\
5 \\
5 \\
5 \\
9\end{array}$ & \begin{tabular}{|l|}
30 \\
30 \\
30 \\
32 \\
30 \\
30 \\
\end{tabular} & $\begin{array}{r}10 \\
8 \\
10 \\
10 \\
10 \\
9\end{array}$ & $\begin{array}{l}0 \\
2 \\
0 \\
0 \\
0 \\
1\end{array}$ & $\mid \begin{array}{l}2 \\
0 \\
2 \\
0 \\
0 \\
0\end{array}$ & $\begin{array}{l}4 \\
3 \\
3 \\
0 \\
0 \\
2\end{array}$ & $\begin{array}{r}6 \\
7 \\
7 \\
10 \\
10 \\
8\end{array}$ & \begin{tabular}{|r|}
10 \\
8 \\
9 \\
3 \\
6 \\
5
\end{tabular} & $\begin{array}{l}0 \\
2 \\
1 \\
7 \\
4 \\
5\end{array}$ & $\begin{array}{l}3 \\
2 \\
1 \\
0 \\
0 \\
1\end{array}$ & $\begin{array}{r}7 \\
8 \\
9 \\
10 \\
10 \\
9 \\
\end{array}$ & $\begin{array}{l}3 \\
1 \\
1 \\
1 \\
1\end{array} \mid$ & \begin{tabular}{r|}
1 \\
0 \\
5 \\
10 \\
7
\end{tabular} & $\begin{array}{r}9 \\
10 \\
5 \\
0 \\
3\end{array}$ & $\begin{array}{l}1 \\
5\end{array}$ & $\begin{array}{l}9 \\
5\end{array}$ \\
\hline
\end{tabular}

blue (tint no. I) -yellow, blue-blue (tint no. I) were given in the order named and, in all but two cases, immediately following the persistence tests. The most striking feature of these results is again the character of the reactions to blue. If brightness were the only factor, a chick trained to black-blue would be expected thereafter to react positively to the less bright of two stimuli, other things being equal, when a different combination were presented. But chicks that one minute accepted black and rejected blue, that is, selected the card of lower brightness value, the next minute accepted white and rejected blue. How shall we explain this behavior if we consider merely the 
brightness values of the stimuli? After black-blue training the ehicks quite commonly rejected this blue no matter with what other color it was in combination. It may be suggested that after the long period of training the chicks respond to a particular brightness value, the blue amounting to a certain degree of gray. But no. 32 and no. 33 rejected blue (tint no. I) when it was used in combination with the much brighter yellow.

The method of training with the electric shock frequently makes the stimulus to which it is attached the emphatic one. In a word, the chicks thus trained may not be guided in their reactions by the color through which they escape, but by the color in connection with which they are shocked. This reaction is one primarily of rejecting blue, rather than of accepting black. It is indicated by such reactions as those of nos. 32,33 , and 39 to black-white after having been trained to black-blue. When white replaced the blue of the original training combination, the stimuli became almost indifferent to the chicks. Again, the same chicks seemed indifferent to the color presented with blue. When required to react to blue-white there was no hesitancy, the response being one of getting-away-from-the-blue-no-matter-what-the-other-color. For data see table 24 .

All the chicks did not behave so, however. A comparison of the corresponding reactions of nos. 17,18 , and 20 is instructive. These animals, when white replaced blue as above, responded definitely and positively to black, and were quite lost when white accompanied blue. With these chicks black seemed to be the guiding stimulus.

It is sometimes said that negation is affirmation, but is this true in any proper psychological sense? Naturally, avoidance of one object means the acceptance of some other object-in the sense that leaving one place means going somewhere else. But does the "somewhere else" need to be defined as a constituent element of the avoiding reaction? Or, upon the occurrence of a positive response, is it necessary to consider the point of departure, which is objectively definable, of course, as a part of the process? The point of departure in the latter or of arrival in the former case does not appear to be essential.

Passing now from the standpoint of stimulus to that of reaction, why should a positive response be regarded as a simultaneous negative response? As antagonists may they not mutu- 
ally exclude each other? But only in so far as a positive reaction essentially includes a negative one and vice versa, is affirmation psychologically negation.

There still remains another aspect of the relation between rejecting and accepting, their possible necessary concomitance. But if, with innervation of a given motor center, there is, according to the Muensterbergian action theory, a simultaneous inhibition of the antagonistic motor center, we are bound to think of real affirmation and real negation as distinct functions, for inhibition is not negation.

What, then, shall we say of our method as a "discrimination method?" From the point of view of behavior discrimination is selective reaction. In this sense the Yerkes method is a discrimination method.

It is interesting to observe the result when neither black nor blue are in the test combination presented after the training. No. 44 on May 22 completed the black-blue habit with three errorless series. On June 6 its persistence test was black, Io; blue, $\circ$. The same day its preference tests on orange-white resulted 5-5. The daily training on orange-white, continued from this date, progressed as follows: $7-3,8-2,7-3$, Io-0, ro-0, Io-0. No. 43, the same age, began black-blue training on the same date as no. 44 and completed the work the day after no. 44 with three perfect series. Its persistence test on June 6 resulted also I0-o. But when tested on the same day for its orange-white preference prior to the training on this combination, its record was orange, Io, white, o. The figures thereafter were $9^{-I}, 9^{-I}, 9^{-I}$, IO-O, IO-O, IO-O. It is apparent in both these cases that the black-blue training strikingly predisposed the chicks to react positively to the darker of the two stimuli in the second combination. It cannot be urged in regard to no. 44 that, either on account of ready modifiability or an easy combination, this was simply a case of rapid learning unassisted by the previous acquisition, first, because orange-white, as indicated by earlier tests, is not so easy a combination; and secondly; the rate of acquisition by this chick was only average, nine days' training having been required for it to complete the blackblue habit.

The important exception to the general rule for brightness training, namely, that when the color which the chick was 
trained to avoid was presented in combination with a new color, the chick continued its specific avoiding reaction regardless of the relative brightness values of the stimuli, demands, I believe, that color quality as well as intensity be assumed as a determining factor in the reactions of these chicks.

\section{F. The Persistence of Modifications}

Hints of the material about to be presented have necessarily been dropped in the discussion of previous topics. Yerkes ${ }^{1}$ publishes in his work on the dancing mouse a valuable table of results on "Measurements of the Duration of a Habit." Commenting on this data, he says, "It is safe, therefore, to conclude from the results which have been obtained that a white-black or black-white discrimination habit may persist during an interval of from two to eight weeks of disuse, but that such a habit is seldom perfect after more than four weeks." If one should assume that the chicks depended upon the element of brightness only, the results obtained for them in our blackblue tests would be comparable with the black-white and whiteblack records of the mice, provided one allowed for age differences, as well as for the fact that the discrimination, assuming the human standard, was made more difficult for the chicks on account of the smaller difference in the brightness of the two stimuli.

The chicks in these tests (see table 25) were placed in the entrance box in the manner followed in the original experiments and allowed to escape from the apparatus to the cage. The cards were exchanged after each trial, that is, regularly alternated right and left. No shock was given and both exits remained open throughout. There was a noticeable difference in the behavior of the chicks in the apparatus after the interval. Instead of smoothly going to work they usually hesitated a few moments and craned their necks about before starting for an opening. The same kind of phenomenon was noticeable, ordinarily, in the behavior of a previously trained chick when lifted in the hand after an interval of some weeks during which it had not been handled. Whereas the animal had come to submit freely and without struggle in the course of the original training, now, when grasped over the back, it sometimes kicked,

1 Yerkes, R. M.: Op. cit., p. 256. 
chirped, or attempted to make flapping movements with the wings. For the tests of persistence, excitement of this kind was avoided as much as possible.

In estimating the value of the results printed in table 25 , it must be remembered that chicks nos. $32,33,38$, and 39 had I 6 days' size training in the interval. Also, nos. 43 and 44 had orange-white training to perfection between the first and the second persistence tests.

TABLE 25

The Persistence of Habit

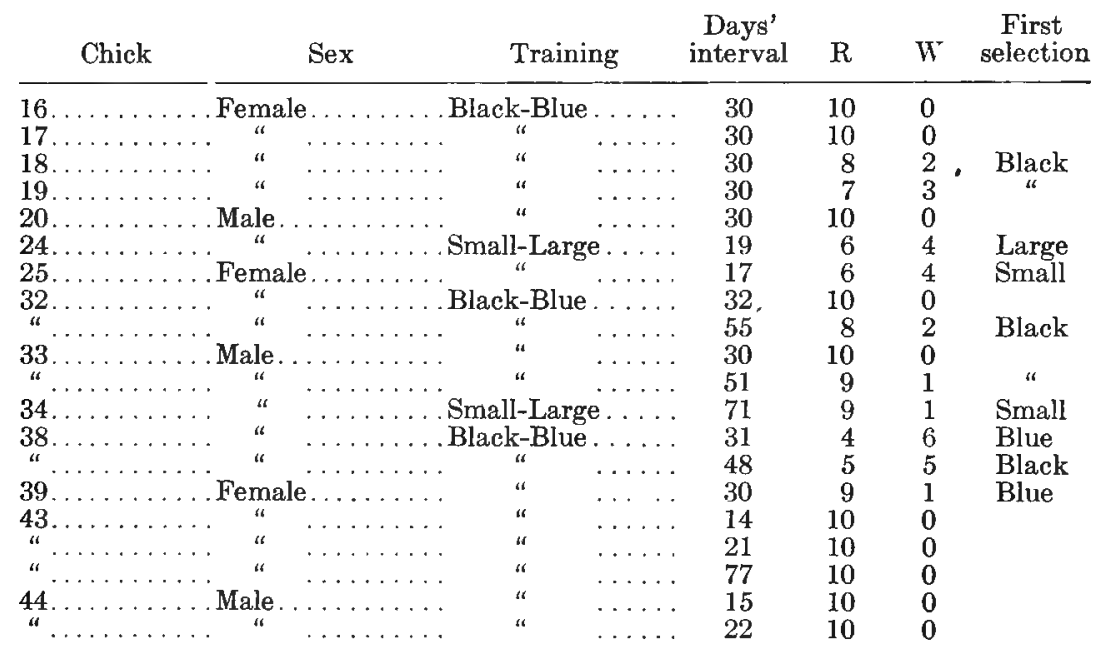

Of eight mice which Yerkes trained in black-white or whiteblack, only two showed perfect persistence of the habit after an interval of four weeks. Of nine chicks trained in black-blue and tested in a similar way after 30 days, five obtained perfect records.

The behavior of no. 34 in the reaction box equipped with the size cards $7 \mathrm{I}$ days after the completion of the original training furnished a nice commentary on the "intelligence" of the anima1. This chick, standing naturally, was $19 \mathrm{~cm}$. high, measured to the highest point of its back. So large that the larger of the two openings would permit its passing out with difficulty, it crouched at the smaller opening and attempted to go through it at every trial but the last. Each time, as soon as it got its head through the small opening and was just beginning to 
struggle, it was brought back to the entrance box for the next trial. The record of its behavior found in table 25 is made subject to the irregularity reported below. The result of this persistence test is the same as that in its last training series. It would be difficult to reach any trustworthy conclusion in regard to the persistence of the small-large habit from the data at hand, for these data have to be considered together with a certain defect in the adjustment of the apparatus. When the persistence tests of nos. 24 and 25 were made, these chicks were so large that they had to squeeze through the smaller opening. For the test of no. 34 the small opening was made slightly larger and a dimension of the larger opening altered to preserve similarity of shape.

TABLE 26

The Rapidity of Modification and its Permanence

Black-Blue Training

\begin{tabular}{|c|c|c|c|c|c|c|c|}
\hline \multirow[b]{2}{*}{ Chick } & \multicolumn{5}{|c|}{ Black-Blue Training } & \multicolumn{2}{|c|}{ Persistence } \\
\hline & Age & Sex & Trials & Errors & Interval & $\mathrm{R}$ & W \\
\hline 16 . & 12 & $\mathrm{~F}$ & 100 & 28 & 30 & 10 & 0 \\
\hline 17. & 12 & $\mathrm{~F}$ & 60 & 20 & 30 & 10 & 0 \\
\hline 18. & 12 & $\mathrm{~F}$ & 100 & 35 & 30 & 8 & 2 \\
\hline 19. & 12 & $\mathrm{~F}$ & 60 & 28 & 30 & 7 & 3 \\
\hline 20 . & 12 & $\mathbf{M}$ & 60 & 19 & 30 & 10 & 0 \\
\hline$\ldots .$. & 12 & $\mathrm{~F}$ & 30 & 10 & 32 & 10 & 0 \\
\hline$\ldots \ldots \ldots \ldots$ & 12 & $\mathbf{M}$ & 70 & 20 & 30 & 10 & 0 \\
\hline$\ldots \ldots \ldots$ & 12 & $\bar{M}$ & 100 & 25 & 31 & 4 & 6 \\
\hline$\ldots \ldots \ldots$ & 12 & $\mathrm{~F}$ & 70 & 20 & 30 & 9 & 1 \\
\hline
\end{tabular}

Index of modifiability ................... 72.2

For the assistance it may be to others in estimating the value of these tests of the persistence of modifications, it should be stated that some of the chicks were in poor physical condition when the tests were made. In the confinement of the laboratory few chicks remained in normal physical condition after eight to twelve weeks.

It is interesting to study the above data on the persistence of habit in connection with the number of trials and errors in the original training series to see if any correlation exists between the rapidity of acquisition and its permanence or retention. In table 26 the necessary items are set side by side. One often hears the pedagogical byword, "Come easy, go easy." This 
certainly does not apply to the chicks tested. Of three animals that required roo trials to perfect the black-blue modification, one made a perfect persistence test; of two that required 70 trials, one made a perfect record; of three that required 60 trials, two made perfect tests; and the chick that completed its work in the shortest time also made a perfect record. The number of trials given in the table is exclusive of preference and final perfect series. In the error column is given for each chick the total number of errors in this number of trials. Of five chicks that made twenty or less errors, four had perfect persistence

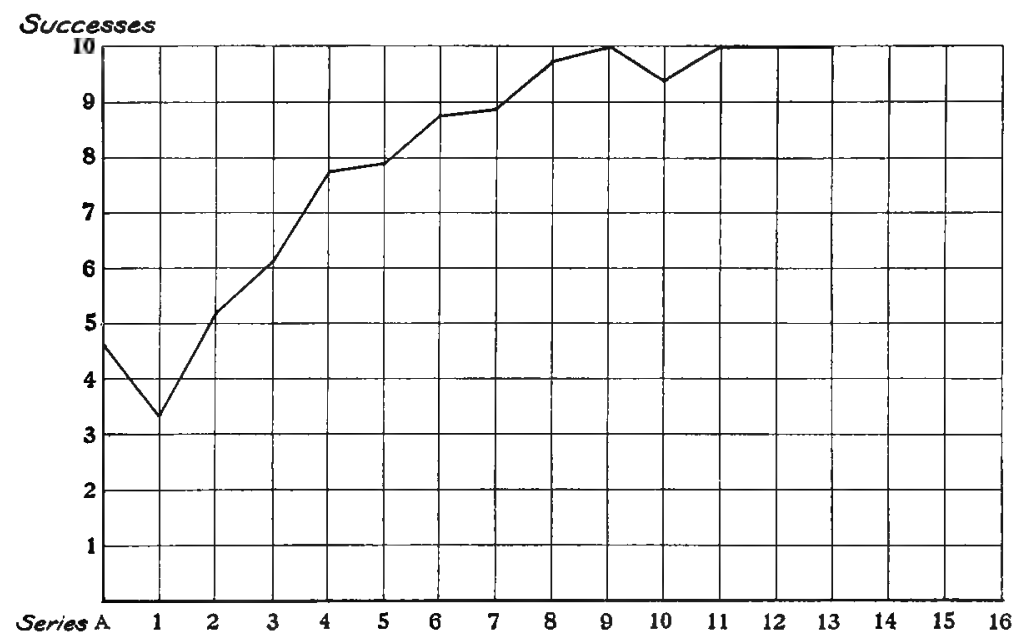

Fraure 11-The success curve for nine chicks of the same age in black-blue training, plotted from averages based on data given in tables 13 and 20. Distances along axis of abscissae represent series; along axis of ordinates, right or successful trials.

tests; of four that made more than twenty errors, one had a perfect persistence test.

One may well question the appropriateness of the expression "trial and error" intended to be descriptive of this method of learning. If the number of errors is included in the number of trials, as for example in this paper, then the original term is awkward if not illogical. These animals learn through successes as well as failures, and the sum of the number of both equals the whole number of trials. In this sense the method is more accurately described as a method of success and failure. Again, 
if the word "trial" in the above expression is meant to refer to the principle of random activity, and " error" to learning based on the avoidance of harmful stimuli, still "trial and error" is an inadequate descriptive term. One could as well say the general law is, "Prove all things; hold fast that which is good."

- If the term selection be construed broadly enough to include both the positive and negative aspects, then trial and selection appears on the whole to be a more satisfactory name for this type of modification.

If the "index of modifiability," to borrow Yerkes' term and definition, be considered "that number of tests after which no errors occur for at least thirty tests," the index of modifiability of these Barred Plymouth Rock chicks, under the conditions described, is 72.2 .

The rate of modification for the same nine chicks is set forth graphically in fig. II. This is a success curve plotted from data in tables $I 3$ and 20 . The averages upon which the curve is based, including the preference test, are as follows: $4.7,3.3$, $5.2,6.1,7.8,7.9,8.8,8.9,9.8,10,9.4$, го, 10, Iо. It is quite possible that valuable accretions to our knowledge of habit and instinct may come through a careful study of the course of development of reactions of both types and a comparison of the quantitative relations involved. I offer the curve of the development of the complete pecking co-ordination (fig. 7) and that of the development of the black-blue modification (fig. II) as suggestions for the beginning of a more specialized investigation.

\section{SUMMARY AND CONCLUSIONS.}

The early post-embryonic life of the chicks continued the scope of activities already begun in the egg. The alternations of passivity and activity, the lifting movements of the head combined with stretching movements of the legs, the occasional reflex forward thrust of the bill followed usually by movements of the mandibles, loud chirping along with other violent activity, -all these were common aspects of the behavior of chicks just before-as well as immediately after hatching. The pecking reaction might have assisted in the process of exclusion, but by far the most common reaction while the chick was struggling in the egg was the lifting movement of the head and bill. This reaction actually broke shell and tore confining membranes. 
The chicks, left to develop naturally in the vicinity of food and water, usually found the water by fortuitous pecking or by performing the drinking movement in imitation of other chicks. By imitation here I mean that the performance of drinking by one chick in the presence of another sometimes stimulated that other itself to perform the drinking act. I reach this conclusion on the basis of incidental observations in the course of the study of other problems. I have no doubt that a special study of this point will bear out the above conclusion. Chicks kept without drink until the third day did not perform the drinking reaction by accident or imitation only. The reaction was elicited by a variety of objects before drink of any kind had touched the bill of the chicks, showing that, when the need of the organism became sufficiently imperative, the drinking reaction appeared in response to many of the same kind of stimuli as the unpracticed pecking reaction. The drinking instinct does not " have to be supplemented by imitation, accident, intelligence, instruction, etc., in order to act." And it is not improbable that the "sight of still water" will be found to evoke this reaction.

The few tests that were made to determine the effect on the pecking reaction of disuse during the first two days of the life of the chick seemed to show (I) that the development of the instinct was retarded by disuse, and (2) that the retardation was quickly overcome with use.

The stimulus for the pecking reaction did not have to be some object of a size convenient for eating. The bill of the chick was used as a testing organ,

Pecking situations were found in which the reaction to one stimulus exerted an inhibitory effect on the reaction to another.

The assumption that chicks do not peck at or eat food when they cannot see it is not supported by the experimental data submitted in this paper. The chicks did peck at and eat food when the light was excluded.

The pecking instinct was investigated primarily to discover the meaning that should attach to such terms as perfection, accuracy, or congenital definiteness, when applied to this response. Pecking is a co-ordination of three reactions,-striking, seizing, and swallowing. The amount of improvement from day to day of the complete co-ordination, not only, but of the com- 
ponents separately, was measured. Records were taken up to the twenty-fifth day. The pecking improved in accuracy very rapidly during the first two days, reaching by the beginning of the third day an efficiency numerically represented by 29.29 on a scale of 50 ; by the beginning of the eleventh day an efficiency of 40 . Io was attained; and during the rest of the period of measurement the degree of accuracy ran no higher than 42.57. The improvement was retarded more by errors in seizing than by errors in swallowing, and more by errors in swallowing than by errors in striking. After the third day the imperfection of seizure remained greater than that of the other two reactions combined. The striking reaction, seldom widely erroneous, improved rapidly and without relapse, closely approximating perfection by the fifth day, - a degree of accuracy that might easily have inspired belief in the perfection of the pecking instinct.

It did not appear that the effect of social influence was such as to increase the rate of improvement in accuracy of the pecking reaction. It may be that the change in the mode of functioning of the organism due to the presence of others was in the direction of increasing the intensity and rapidity of the reactions, without increasing their rate of improvement in accuracy. Such a variation would have selective value, inasmuch as the animal would get more food in a given time, even though the pecking were no more accurate.

The important exception to the general rule for brightness training, namely, that when the color which the chick was trained to avoid was presented in combination with a color other than that used in the original training, the chick continued its specific avoiding reaction regardless of the relative brightness values of the stimuli, demands, I believe, that color quality as well as intensity be assumed as a determining factor in the reactions of the chicks.

The chicks, without much doubt, responded selectively to one of two objects of different size.

The results of the form tests reported were purely negative.

There was no conclusive evidence that previous formation of the black-blue habit facilitated the formation of the small-large habit. 
The tests of retention of the black-blue habit after size training, which intervened between the original training and the retention tests, at least suggest a method that may be useful in studies of the interrelation of habits.

The method of training with the electric shock frequently made the stimulus to which it was attached the emphatic one. In such a case the chicks were not guided in their reactions by the color through which they escaped, but by the color in connection with which they were shocked. That is, the reaction was negative to blue, not positive to black, when this occurred in black-blue training. Psychologically, it seems, negation is not affirmation.

Of nine chicks perfectly trained in black-blue, five made perfect persistence tests after an interval of thirty days.

In the case of the same tests of retention, rapidity of modification was positively correlated with permanence of modification.

For these nine chicks the "index of modifiability" was 72.2 .

"Trial and error", is an unsatisfactory name for this method of learning. 


\section{Behavior Monographs}

\section{Volume 1}

I. The development of certain instincts and habits in chicks. By Frederick S. Breed. Pp. iv+78. \$1.00

\section{In Preparation}

Methods of studying vision in animals. By Robert M. Yerkes and Johin B. Watson.

An experimental study on the death-feigning of Belostoma (-zaithā aucct.) flumineum say and nepa apiculata uhler. By Henry H. P. Severin and Harry C. Severin.

The Biology of Physa. By Jean Dawson. 




